OPEN ACCESS

Edited by: Ruth Grene,

Virginia Tech, USA

Reviewed by:

Erik T. Nilsen,

Virginia Tech, USA

Bishal Gole Tamang,

University of Minnesota, USA

*Correspondence:

Weibiao Liao

liaowb@gsau.edu.cn

Specialty section: This article was submitted to

Plant Abiotic Stress,

a section of the journa

Frontiers in Plant Science

Received: 28 October 2016 Accepted: 20 January 2017

Published: 07 February 2017

Citation:

Chen Y, Wang M, Hu L, Liao W, Dawuda MM and Li C (2017) Carbon

Monoxide Is Involved in Hydrogen

Gas-Induced Adventitious Root Development in Cucumber under

Simulated Drought Stress.

Front. Plant Sci. 8:128.

doi: 10.3389/fpls.2017.00128

\section{Carbon Monoxide Is Involved in Hydrogen Gas-Induced Adventitious Root Development in Cucumber under Simulated Drought Stress}

\author{
Yue Chen, Meng Wang, Linli Hu, Weibiao Liao*, Mohammed M. Dawuda and Chunlan Li \\ College of Horticulture, Gansu Agricultural University, Lanzhou, China
}

Hydrogen gas $\left(\mathrm{H}_{2}\right)$ and carbon monoxide $(\mathrm{CO})$ are involved in plant growth and developmental processes and may induce plant tolerance to several stresses. However, the independent roles and interaction effect of $\mathrm{H}_{2}$ and $\mathrm{CO}$ in adventitious root development under drought conditions have still not received the needed research attention. We hypothesize that there exists crosstalk between $\mathrm{H}_{2}$ and $\mathrm{CO}$ during adventitious root development under drought stress. The results of our current study revealed that $50 \%(\mathrm{v} / \mathrm{V})$ hydrogen-rich water $(\mathrm{HRW}), 500 \mu \mathrm{M}$ Hemin (the CO donor) and $30 \%$ (w/v) CO aqueous solution apparently promoted the development of adventitious roots in cucumber explants (Cucumis Sativus L.) under drought stress. $\mathrm{H}_{2}$ and $\mathrm{CO}$ increased relative water content (RWC), leaf chlorophyll content (chlorophyll $\mathrm{a}, \mathrm{b}$, and $\mathrm{a}+\mathrm{b}$ ), and chlorophyll fluorescence parameters [photochemical efficiency of photosystem II (PSII), PSII actual photochemical efficiency and photochemical quench coefficient] under drought condition. When the CO scavenger hemoglobin $(\mathrm{Hb})$ or zinc protoporphyrin IX (ZnPPIX) was added to HRW/CO aqueous solution, the positive effect of HRW/CO aqueous solution on RWC, leaf chlorophyll content, and chlorophyll fluorescence parameters were reversed. Additionally, superoxide dismutases, peroxidase, catalase, and ascorbate peroxidase was significantly increased in the explants treated with $\mathrm{HRW}$ and $\mathrm{CO}$ aqueous solution under drought stress, thus alleviating oxidative damage, as indicated by decreases in thiobarbituric acid reactive substances (TBARS), hydrogen peroxide $\left(\mathrm{H}_{2} \mathrm{O}_{2}\right)$, and superoxide radical $\left(\mathrm{O}_{2}^{-}\right)$levels. $\mathrm{H}_{2}$ and $\mathrm{CO}$ also improved the levels of water soluble carbohydrate, total soluble protein, and proline content. However, the above $\mathrm{CO} / \mathrm{H}_{2}$-mediated effects were reversed by $\mathrm{CO}$ scavenger $\mathrm{Hb}$ or $\mathrm{CO}$ specific synthetic inhibitor ZnPPIX. Therefore, $\mathrm{CO}$ may be involved in $\mathrm{H}_{2}$-induced adventitious rooting under drought stress and alleviate oxidative damage by enhancing RWC, leaf chlorophyll content, chlorophyll fluorescence parameters, metabolic constituent content, activating anti-oxidant enzymes and reducing TBARS, $\mathrm{O}_{2}^{-}$, and $\mathrm{H}_{2} \mathrm{O}_{2}$ levels.

Keywords: adventitious rooting, drought, HRW, CO, chlorophyll fluorescence, enzyme activity 


\section{INTRODUCTION}

Drought stress which is among the important abiotic stresses that adversely affects plant growth and development has become a global problem (Farooq et al., 2009). In order to withstand drought, plants develop multiple defense mechanisms. Recently, many studies have focused on understanding adventitious rooting response signaling pathways under drought stress. Adventitious root formation is a very complex process. Enzymes such as peroxidase (POD), polyphenol oxidase (PPO), and indoleacetic acid oxidase (IAAO) are known to be intimately involved in the process of adventitious root formation (Smart et al., 2003). This process is affected by factors such as temperature, light conditions, water, and nutrient supply (Takahashi et al., 2003). Among plant hormones, auxins are of vital importance, because they can promote the formation of primordial roots. Recent studies found signal molecules that regulate in auxin-induced adventitious root development, for instance hydrogen peroxide $\left(\mathrm{H}_{2} \mathrm{O}_{2}\right.$, Liao et al., 2012; Niu and Liao, 2016), nitric oxide (NO, Zhu et al., 2016b), carbon monoxide (CO, Wang and Liao, 2016), hydrogen sulfide $\left(\mathrm{H}_{2} \mathrm{~S}\right.$, Lin et al., 2012), and calcium $\left(\mathrm{Ca}^{2+}\right.$, Cui et al., 2015). To date, however, the intricate signaling network that participates in adventitious root development remains unresolved.

Hydrogen gas $\left(\mathrm{H}_{2}\right)$ is colorless, odorless, and tasteless, and is considered to be physiologically inert molecule. $\mathrm{H}_{2}$ was considered to be a selective reductant (Ohsawa et al., 2007) and be involved in the development and stress responses in plants (Renwick et al., 1964; Zeng et al., 2013). Hydrogen-rich water (HRW) treatment could delay postharvest ripening and senescence of kiwifruit by reducing the rot incidence, inhibiting the respiration intensity, and decreasing the lipid perxidation level postharvest fruits ( $\mathrm{Hu}$ et al., 2014). $\mathrm{H}_{2}$ enhanced Arabidopsis salt tolerance by modulating genes/proteins of zinc-finger transcription factor ZAT10/12 (Xie et al., 2012). Exogenous HRW effectively led to an increase of intracellular $\mathrm{H}_{2}$ production, and a reduction in the stomatal aperture, and therefore resulted in enhanced drought tolerance (Xie et al., 2014). Wu et al. (2015) found that 50\% HRW significantly alleviated the cadmium toxic symptoms by increasing the antioxidant capacities in Chinese cabbage. HRW conferred tolerance to UVB-induced oxidative damage partially by the manipulation of (iso)flavonoids metabolism in Medicago sativa L. (Xie et al., 2015). HRW significantly blocked UV-A-induced the accumulation of $\mathrm{H}_{2} \mathrm{O}_{2}$ and $\mathrm{O}_{2}$ ( $\mathrm{Su}$ et al., 2014). Results of previous studies have shown that $\mathrm{H}_{2}$ promoted adventitious root development in a CO-dependent manner (Lin et al., 2014). During adventitious rooting, $\mathrm{H}_{2}$ mediated target genes related to auxin signaling and adventitious root development, CsDNAJ-1, CsCPDK1/5, CsCDC6, CsAUX228like, and CsAUX22D-like through CO pathways. Our results also confirmed that $\mathrm{H}_{2}$ promoted adventitious rooting by increased the content of $\mathrm{NO}$ and the activities of NO synthase and nitrate reductase (Zhu et al., 2016b). Additionally, $\mathrm{H}_{2}$ activated cell cycle and up-regulated cell cycle-related genes and adventitious rooting-related genes via NO pathway (Zhu et al., 2016a).

Carbon monoxide is one of the most important reactive trace gases in nature, which has long been widely considered as a poisonous gas. Siegel et al. (1962) reported that germinating seeds of rye (Secale cereale), pea (Pisum sativum), cucumber, and lettuce (Lactuca sativa) produced $\mathrm{CO}$ at levels of 10 to $25 \mathrm{~mL} \mathrm{~L}^{-1}$. Piantadosi (2002) found that CO influenced cell proliferation and the production of cytokines in plants. $\mathrm{CO}$ is bioactive molecule involved in many biological processes. It has been reported that $\mathrm{CO}$ played a major role in mediating the cytoprotection against oxidant-induced lung injury and neurotransmission (Huang et al., 2006). In addition, CO could acts as a signal molecule involved in plant growth and development which requires the participation of other signaling molecules. For instance, CO delayed gibberellins (GA)-triggered programmed cell death (PCD) in wheat aleurone cells by up-regulating of ascorbate peroxidase (APX) and catalase (CAT) expression, and decreasing $\mathrm{H}_{2} \mathrm{O}_{2}$ overproduction ( $\mathrm{Wu}$ et al., 2010). Low CO concentrations $(0.1$ or $1 \%)$ in air stimulated seed germination in dormant giant foxtail (Setaria faberi; Dekker and Hargrove, 2002). CO produced by heme oxygenase might be involved in ABAinduced stomatal closure in Vicia faba (Cao et al., 2007). $\mathrm{CO}$ enhanced antioxidant defense system in abiotic stresses tolerance, such as drought (Liu et al., 2010), salt (Ling et al., 2009), ultraviolet radiation (Yannarelli et al., 2006), and heavy metal (Meng et al., 2011). Xuan et al. (2008) found that $\mathrm{CO}$ induced adventitious rooting in dose- and timedependent manners. The application of $\mathrm{CO}$ aqueous solution was able to alleviate the IAA depletion-induced inhibition of adventitious rooting. Methane-rich water stimulated adventitious rooting, which was partially mediated by $\mathrm{CO}$ in cucumber (Cui et al., 2015). Moreover, CO showed cross talk with other signaling molecules including $\mathrm{NO}$, phytohormones (IAA, ABA, and $\mathrm{GA}$ ), $\mathrm{H}_{2} \mathrm{~S}, \mathrm{H}_{2}$, and $\mathrm{CH}_{4}$ (Wang and Liao, 2016).

$\mathrm{H}_{2}$ and $\mathrm{CO}$ as new gas signaling modulators are involved in signal path ways of plants. Studies have shown the involvement of heme oxygenase-1 (HO-1, a novel antioxidant enzyme)/CO in $\mathrm{H}_{2}$-induced osmotic stress tolerance in alfalfa (Jin et al., 2016). In addition, $\mathrm{H}_{2}$ mediated target genes related to auxin signaling and adventitious root development through $\mathrm{CO}$ pathways during adventitious rooting (Lin et al., 2014). As mentioned above, both $\mathrm{H}_{2}$ and $\mathrm{CO}$ could act as important gaseous molecules with multiple biological functions in plant response against abiotic stress. However, the signal roles of $\mathrm{H}_{2}$ and $\mathrm{CO}$ under abiotic stress during adventitious rooting remain unclear. It may be hypothesized that $\mathrm{H}_{2}, \mathrm{CO}$, and their crosstalk could be involved in adventitious root development in plants under drought stresses. Therefore, the present study focuses on the effect of $\mathrm{H}_{2}$ and $\mathrm{CO}$ on the adventitious root development in cucumber under drought stress and their relationship during that process. The results presented in this work are significant for both fundamental and applied plant biology. 


\section{MATERIALS AND METHODS}

\section{Plant Materials}

Cucumber seeds (Cucumis Sativus 'Xinchun No. 4') were purchased from the Gansu Academy of Agricultural Sciences in Lanzhou, China. The seeds were surface sterilized in 5\% (w/v) sodium hypochlorite for $10 \mathrm{~min}$ and then soaked in distilled water for $5 \mathrm{~h}$. The seeds were germinated on filter paper with distilled water in Petri dishes $(15 \mathrm{~cm}$-diameter, $2.5 \mathrm{~cm}$ deep) and maintained at $25 \pm 1^{\circ} \mathrm{C}$ for 7 days with a 14 -h photoperiod (photosynthetically active radiation $=200 \mu \mathrm{mol}$ $\mathrm{m}^{-2} \mathrm{~s}^{-1}$ ). The 7-day-old cucumber seedlings with primary roots removed were used as explants and maintained under the same temperature and photoperiod conditions for another 7 days in the presence of different media as indicated below. The number of adventitious roots per explant was counted and recorded.

\section{Experimental Treatments}

All the chemicals used in the experiments were obtained from Sigma (St. Louis, MO, USA) unless otherwise stated. Drought stress was simulated by application of polyethylene glycol 6000 (PEG; obtained from Shanghai Chemical Reagent Co. Ltd. Shanghai, China). Based on previous reports, we used different concentrations of PEG (0.1, 0.3, 0.6, and $0.9 \%, \mathrm{~m} / \mathrm{v})$, HRW $(10,30$, and 50\%, v/v; Xie et al., 2014), the CO donor Hemin $(10,100,500$, and $1000 \mu \mathrm{M})$ and $\mathrm{CO}$ aqueous solution $(10,30$, and $50 \%$, w/v; Xuan et al., 2008) as indicated in Figure 1 and kept at $25 \pm 1^{\circ} \mathrm{C}$. The compound zinc protoporphyrin IX (ZnPPIX, Sigma) was used at $100 \mu \mathrm{mol} \mathrm{L^{-1 }}$ as a specific inhibitor of CO. Hemoglobin ( $\mathrm{Hb}$, Sigma) was chosen as $\mathrm{CO}$ scavenger at $0.5 \mathrm{~g} \mathrm{~L} \mathrm{~L}^{-1}$ (Xuan et al., 2008). Seven days old explants which were treated distilled water throughout the experiment served as control to provide a basis to compare the effects of $\mathrm{H}_{2}$ and $\mathrm{CO}$ under drought conditions. The experiment included nine treatments: (1) Con $\rightarrow$ Con (the control; Con) explants treated with distilled water for 7 days. (2) Con $\rightarrow$ PEG (PEG treatment), explants pretreated with distilled water for 2 days and then transferred to PEG solution for 5 days. (3) $\mathrm{HRW} \rightarrow$ PEG (HRW treatment), explants pretreated with HRW for 2 days and then transferred to PEG solution for 5 days. (4) $\mathrm{CO} \rightarrow$ PEG (CO treatment), explants pretreated with $\mathrm{CO}$ aqueous solution for 2 days and then transferred to PEG solution for 5 days. (5) $\mathrm{CO}+\mathrm{HRW} \rightarrow \mathrm{PEG} \quad(\mathrm{CO}+\mathrm{HRW}$ treatment), explants pretreated with CO+HRW for 2 days and then transferred to PEG solution for 5 days. (6) $\mathrm{HRW}+\mathrm{Hb} \rightarrow \mathrm{PEG}$ (HRW $+\mathrm{Hb}$ treatment), explants pretreated with $\mathrm{HRW}+\mathrm{Hb}$ for 2 days and then transferred to PEG solution for 5 days. (7) HRW + ZnPPIX $\rightarrow$ PEG (HRW +ZnPPIX treatment), explants pretreated with HRW+ZnPPIX for 2 days and then transferred to $\mathrm{PEG}$ solution for 5 days. (8) $\mathrm{CO}+\mathrm{Hb} \rightarrow \mathrm{PEG}(\mathrm{CO}+\mathrm{Hb}$ treatment), explants pretreated with $\mathrm{CO}+\mathrm{Hb}$ for 2 days and then transferred to PEG solution for 5 days. (9) CO $+\mathrm{ZnPPIX} \rightarrow \mathrm{PEG}$ $(\mathrm{CO}+\mathrm{ZnPPIX}$ treatment), explants pretreated with $\mathrm{CO}+\mathrm{ZnPPIX}$ for 2 days and then transferred to PEG solution for 5 days. The concentration of these chemicals was selected based on the results of a preliminary experiment. The treatments were arranged in a completely randomized design with at least three replications.

\section{Leaf Relative Water Content}

Leaf relative water content (RWC) was measured as described by Aroca et al. (2003) after 0, 12, 24, and $48 \mathrm{~h}$ of PEG treatment. One leaf samples per explants were immediately weighed (fresh weight) then placed in a water vapor-saturated vial for $24 \mathrm{~h}$ and weighed (turgid weight). The samples were dried in an oven at $80^{\circ} \mathrm{C}$ for $48 \mathrm{~h}$ and their dry weights (dry weight) were determined. At each point in time, three replicates were performed. RWC was calculated by the following equation: [(fresh weight - dry weight)/(turgid weight - dry weight) $] \times 100$.

\section{Leaf Chlorophyll Content and Leaf Chlorophyll Fluorescence}

To determined chlorophyll content, $0.5 \mathrm{~g}$ leaves were frozen in liquid nitrogen, grounded to a powder, and extracted in $5 \mathrm{ml}$ of $80 \%(\mathrm{v} / \mathrm{v})$ acetone until complete bleaching. Chlorophyll was quantified by measuring the absorption at 645 and $663 \mathrm{~nm}$ and its concentration was calculated according to Arnon (1949). Chlorophyll content was expressed in $\mathrm{mg} \cdot \mathrm{g}^{-1}$ fresh weight. Chlorophyll fluorescence was measured with modulated chlorophyll fluorescence imaging system ImagingPAM (Walz, Effeltrich, Germany). Chl fluorescence parameters were calculated using the following equations: maximal quantum yield of photosystem II (PSII), Fv/Fm; quantum efficiency of PSII electron transport, $\Phi$ PS II $=\left(F \mathrm{~m}^{\prime}-F \mathrm{~s}\right) / F^{\prime}$; photochemical quenching, $\mathrm{qP}=\left(F \mathrm{~m}^{\prime}-F \mathrm{~s}\right) / F \mathrm{v}^{\prime}$. The seedlings leaves were kept in darkness for $30 \mathrm{~min}$ to allow all reaction centers to open. Chl content and chl fluorescence were measured after $0,12,24$, and $48 \mathrm{~h}$ of PEG treatment, respectively.

\section{Determination of TBARS, $\mathrm{O}_{2}{ }^{-}$, and $\mathrm{H}_{2} \mathrm{O}_{2}$}

The level of lipid peroxidation was measured in terms of thiobarbituric acid reactive substances (TBARS) concentration (Heath and Packer, 1968). Fresh leaf samples (0.2 g) were homogenized in $10 \mathrm{~mL}$ of $0.1 \%$ trichloroacetic acid (TCA). The homogenate was centrifuged at $1699 \times g$ for $10 \mathrm{~min}$. About $2 \mathrm{~mL}$ of aliquot of the supernatant was mixed with $4 \mathrm{~mL}$ of $0.5 \%$ TBA in $20 \%$ TCA. The mixture was heated at $95^{\circ} \mathrm{C}$ for $20 \mathrm{~min}$ and then quickly cooled in an ice bath. After centrifugation at $10621 \times g$ for $10 \mathrm{~min}$ to remove the suspended turbidity, the absorbance of the supernatant was recorded at 523, 600, and $450 \mathrm{~nm}$, respectively. The TBARS concentration was calculated using its absorption coefficient $\left(155 \mathrm{mM}^{-1} \mathrm{~cm}^{-1}\right)$.

Superoxide radical $\left(\mathrm{O}_{2}{ }^{-}\right)$level was measured as described by Elstner and Heupel (1976) with some modifications. One gram of frozen leaf segments was homogenized with $3 \mathrm{ml}$ of $65 \mathrm{mM}$ potassium phosphate buffer $(\mathrm{pH} 7.8)$ and centrifuged at $2655 \times g$ for $10 \mathrm{~min}$. The incubation mixture contained $0.9 \mathrm{ml}$ of $65 \mathrm{mM}$ phosphate buffer ( $\mathrm{pH} 7.8$ ), $0.1 \mathrm{ml}$ of $10 \mathrm{mM}$ hydroxylamine 


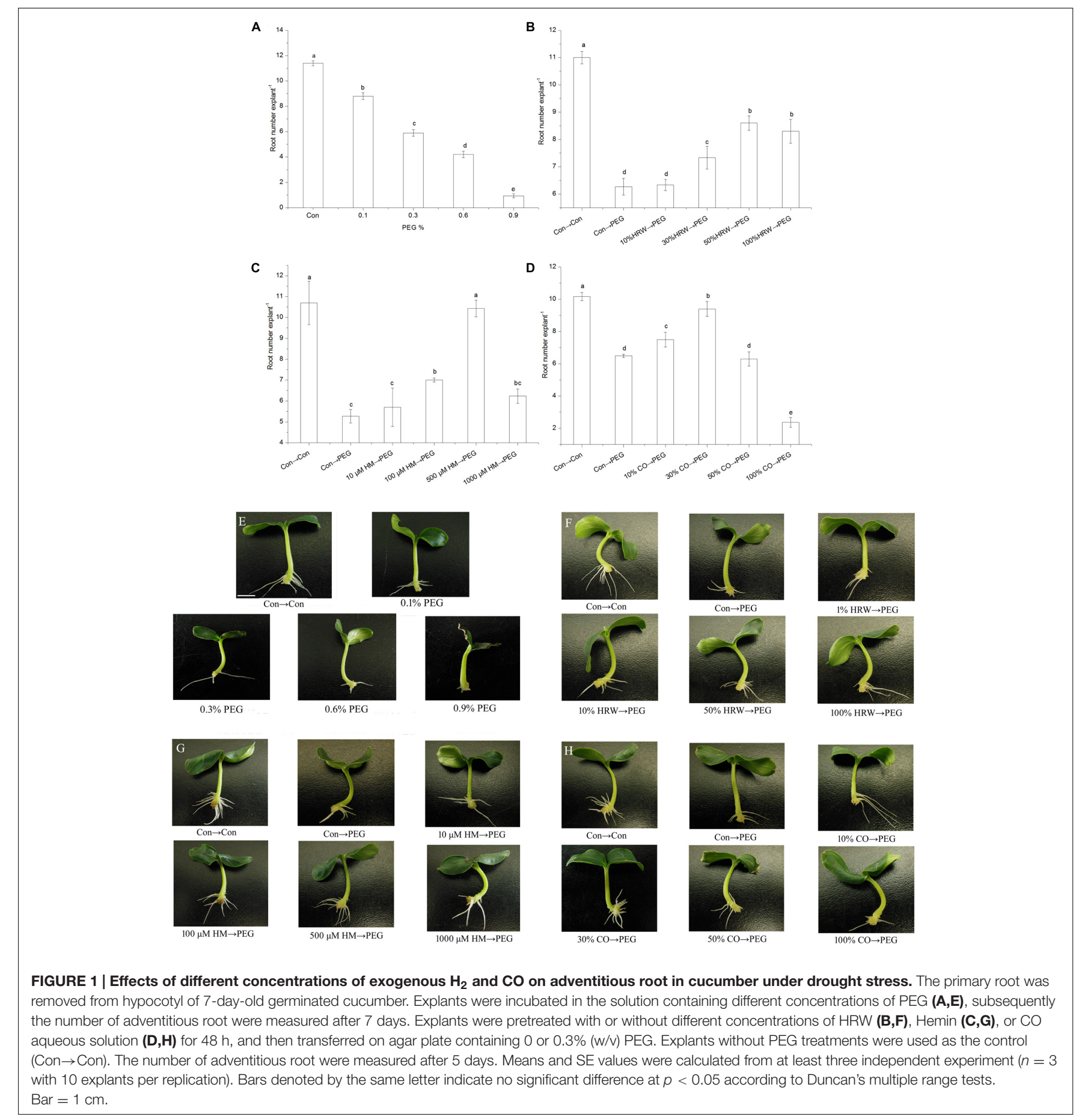

hydrochloride, and $1 \mathrm{ml}$ of the supernatant. After incubation at $25^{\circ} \mathrm{C}$ for $20 \mathrm{~min}, 17 \mathrm{mM}$ sulfanilamide and $7 \mathrm{mM}$-naphthylamine were added to the incubation mixture. After reaction at $25^{\circ} \mathrm{C}$ for $20 \mathrm{~min}$, ethyl ether in the same volume was added and centrifuged at $1699 \times g$ for $5 \mathrm{~min}$. The content of $\mathrm{O}_{2}{ }^{-}$was estimated by measuring the spectrum absorbance of the supernatant at $530 \mathrm{~nm}$ and using a standard curve plotted with a known concentration of $\mathrm{NO}_{2}{ }^{-}$.
$\mathrm{H}_{2} \mathrm{O}_{2}$ content in cucumber explants was determined according to Mukherjee and Choudhuri (1983) with some modifications. Cucumber explants $(0.2 \mathrm{~g})$ were homogenized in an ice bath with $2 \mathrm{~mL}$ of $0.1 \%(\mathrm{w} / \mathrm{v})$ TCA. The homogenate was centrifuged at $1699 \times g$ for $10 \mathrm{~min}$ and $0.5 \mathrm{~mL}$ of the supernatant was added to $0.5 \mathrm{~mL}$ of $10 \mathrm{mM}$ potassium phosphate buffer ( $\mathrm{pH} 7.0$ ) and $1 \mathrm{~mL}$ of $1 \mathrm{M} \mathrm{KI}$. The content of $\mathrm{H}_{2} \mathrm{O}_{2}$ was estimated by measuring the spectrum absorbance of the supernatant 
at $415 \mathrm{~nm}$ and using a standard curve plotted with a known concentration of $\mathrm{H}_{2} \mathrm{O}_{2}$.

\section{Antioxidant Enzymes Assays}

The supernatants used for assays of enzyme activity were prepared as described by Jin et al. (2013) with some modifications. Total superoxide dismutases (SOD) activity was assayed by measuring its capacity of inhibiting the photochemical reduction of Nitrotetrazolium Blue chloride (NBT). One unit of SOD (U) was defined as the amount of crude enzyme extract required to inhibit the reduction rate of NBT by $50 \%$. POD was determined by measuring the oxidation of guaiacol (extinction coefficient $26.6 \mathrm{mM}^{-1} \mathrm{~cm}^{-1}$ ) at $470 \mathrm{~nm}$. CAT activity was determined by following the consumption of $\mathrm{H}_{2} \mathrm{O}_{2}$ (extinction coefficient $39.4 \mathrm{mM}^{-1} \mathrm{~cm}^{-1}$ ) at $240 \mathrm{~nm}$ for $3 \mathrm{~min}$. The reaction mixture contained $0.025 \mathrm{~mol} \mathrm{~L}^{-1}$ potassium phosphate buffer ( $\mathrm{pH} 7.0$ ), $20 \mathrm{mM} \mathrm{H}_{2} \mathrm{O}_{2}$ and $0.1 \mathrm{ml}$ of enzyme extract in a $3 \mathrm{ml}$ volume. APX activity was determined by monitoring the decrease at $290 \mathrm{~nm}$ (extinction coefficient $\left.2.8 \mathrm{mM}^{-1} \mathrm{~cm}^{-1}\right)$.

\section{Osmotic Regulators Content Estimation}

Water soluble carbohydrate was determined after $0,12,24$, and $48 \mathrm{~h}$ of PEG treatment using the anthrone method as described by Liao et al. (2012) with some modifications. Water soluble carbohydrate was extracted for $12 \mathrm{~h}$ at $70^{\circ} \mathrm{C}$ after the explants samples $(0.2 \mathrm{~g})$ had been homogenized. The carbohydrate extract was analyzed by reacting $0.5 \mathrm{~mL}$ of the supernatant with $0.5 \mathrm{~mL}$ of freshly prepared anthrone reagent ( $1 \mathrm{~g}$ anthrone in $50 \mathrm{~mL}$ ethyl acetate), $1.5 \mathrm{~mL}$ distilled $\mathrm{H}_{2} \mathrm{O}$ and $5 \mathrm{~mL}$ of concentrated sulfuric acid, and then placing it in boiling water bath for $1 \mathrm{~min}$. After cooling to room temperature, the absorbance at $630 \mathrm{~nm}$ was measured. Total soluble protein content was measured according to the procedure described by Bradford (1976), using bovine serum albumin as a calibration standard. The soluble protein fraction was obtained after centrifugation of the crude extract at $1699 \times g$ and $4^{\circ} \mathrm{C}$ for $10 \mathrm{~min}$.

Determination of proline content was done according to Bates et al. (1973) with some modifications. Cucumber explants $(0.2 \mathrm{~g})$ from each group were homogenized in $3 \%(\mathrm{w} / \mathrm{v})$ sulphosalicylic acid and homogenate filtered through filter paper. After addition of acid ninhydrin and glacial acetic acid, the resultant mixture was heated at $100^{\circ} \mathrm{C}$ for $1 \mathrm{~h}$ in water bath. Reaction was then stopped by using ice bath. The mixture was extracted with toluene, and the absorbance of fraction with toluene aspired from liquid phase was read at $520 \mathrm{~nm}$.

\section{Statistical Analysis}

Results were expressed as the mean values \pm SE of three independent experiments $(n=3)$. In the determination of root number, 10 cuttings were used per replication. In the determination of the physiological and biochemical indicators, we used different weight sample per replication which were mentioned in the assay methods. Statistical analysis was performed using software SPSS 17.0. Analysis of Variance
(ANOVA) was done and the Duncan's multiple range test $(P<0.05)$ was used in separating treatment means which showed significant differences from the analysis of variance conducted.

\section{RESULTS}

\section{Effect of Exogenous Pretreatments with $\mathrm{H}_{2}$ and $\mathrm{CO}$ on Adventitious Root Development under Simulated Drought Stress}

As shown in Figure 1, the number of adventitious root significantly decreased with the increase of PEG concentration. Compared with the control (Con), the root number of 0.1, $0.3,0.6$, and $0.9 \%$ PEG treated explants decreased by 22.81 , $48.25,63.16$, and $91.84 \%$, respectively. Therefore, treatments with $0.1 \%$ PEG, $0.3 \%$ PEG, and 0.6-0.9\% PEG could be termed as low, moderate, and severe drought stress, respectively. The $0.3 \%$ PEG was used to simulate moderate drought in the subsequent experiments.

To study the effects of exogenous $\mathrm{H}_{2}$ and $\mathrm{CO}$ on adventitious root development under drought stress, we performed doseresponse experiments with HRW, the CO donor Hemin and CO aqueous solution. When compared with the control explants, treatment with PEG significantly decreased adventitious root number (Figure 1). Explants pretreated with 30, 50, and 100\% HRW had a significant increase in root number compared with PEG-treated explants, indicating that PEG-induced drought stress was alleviated by HRW. Among the various HRW concentrations, 50\% HRW promoted rooting better than the other treatments (Figure 1).

As shown in Figure 1, pretreatments with different concentrations of Hemin (100-1000 $\mu \mathrm{M})$ increased adventitious root number under drought stress compared with PEG treatment. The maximum root number was observed in the $500 \mu \mathrm{M}$ Hemin treatment, reached the level of the control. Figure 1 showed that 10 and $30 \% \mathrm{CO}$ aqueous solution pretreatments resulted in increased root number under drought stress. However, 50 and $100 \% \mathrm{CO}$ aqueous solution pretreatments decreased root number under drought stress. Therefore, $500 \mu \mathrm{M}$ Hemin and 30\% CO aqueous solution which were the most effective under our experimental conditions were used for further studies.

\section{Effect of Pretreatments with $\mathrm{Hb}$ and ZnPPIX on Adventitious Root Development under Simulated Drought Stress}

To understand the interaction effect of $\mathrm{H}_{2}$ and $\mathrm{CO}$, we studied the effects of $\mathrm{CO}$ scavenger or inhibitor on $\mathrm{H}_{2}$-induced adventitious rooting under drought stress. As shown in Figure 2, HRW, CO, and $\mathrm{CO}+\mathrm{HRW}$ increased root number under drought stress. The HRW/CO-induced adventitious rooting under drought condition was reserved by $\mathrm{CO}$ scavenger $\mathrm{Hb}$ and inhibitor 


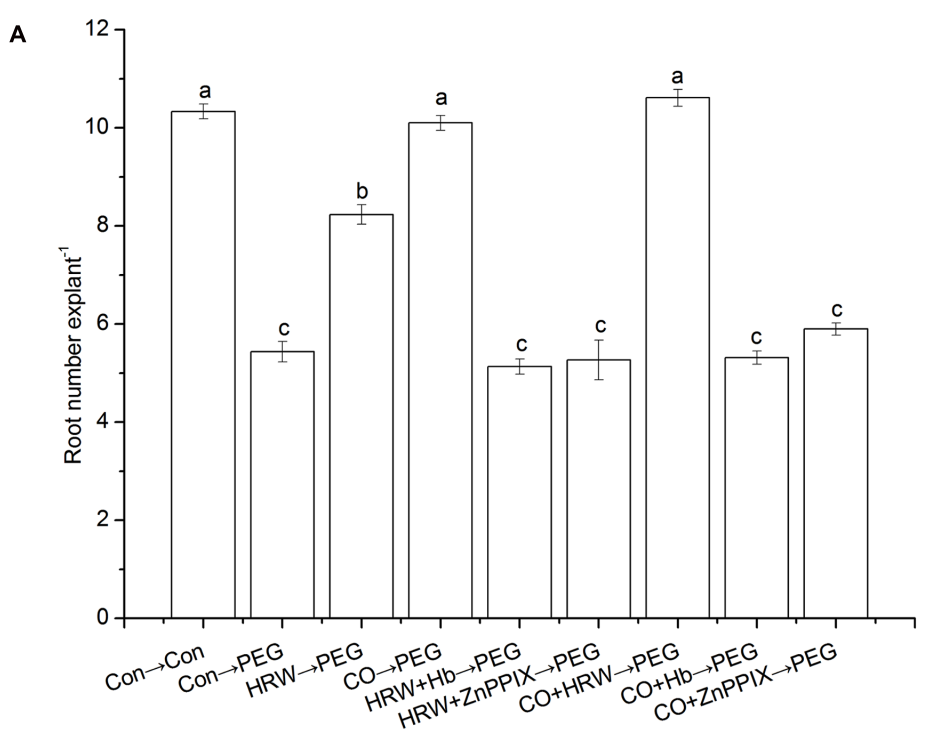

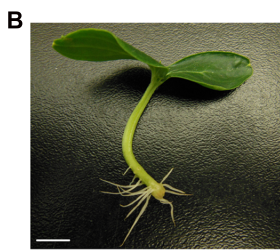

Con $\rightarrow$ Con

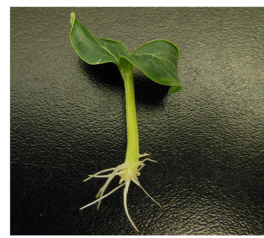

$\mathrm{CO} \rightarrow \mathrm{PEG}$

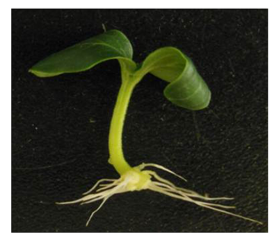

$\mathrm{CO}+\mathrm{HRW} \rightarrow \mathrm{PEG}$

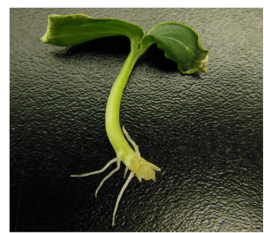

Con $\rightarrow$ PEG

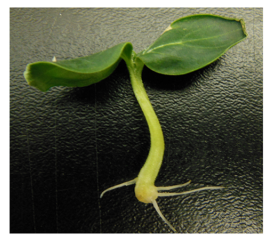

$\mathrm{HRW}+\mathrm{Hb} \rightarrow \mathrm{PEG}$

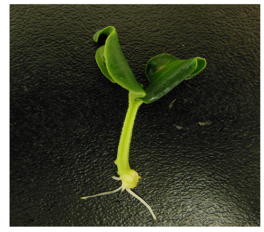

$\mathrm{CO}+\mathrm{Hb} \rightarrow \mathrm{PEG}$

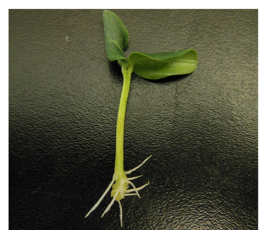

$\mathrm{HRW} \rightarrow \mathrm{PEG}$

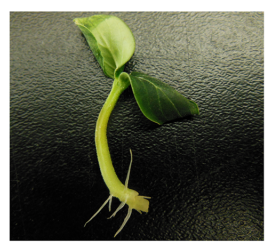

$\mathrm{HRW}+\mathrm{ZnPPIX} \rightarrow \mathrm{PEG}$

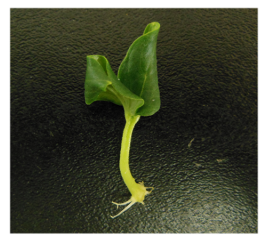

$\mathrm{CO}+\mathrm{ZnPPIX} \rightarrow \mathrm{PEG}$

FIGURE 2 | Effect of $\mathrm{CO}$ scavenger $\mathrm{Hb}$ or the CO-specific synthetic inhibitor ZnPPIX on adventitious root in cucumber explants under drought stress. The primary root was removed from hypocotyls 7-day-old germinated cucumbers. Explants were pretreated with or without Hb or ZnPPIX for $48 \mathrm{~h}$, and then transferred on agar plate containing 0 or $0.3 \%$ (w/v) PEG. Explants without PEG treatments were used as the control (Con $\rightarrow$ Con). Means and SE values (A) were calculated from at least three independent experiment ( $n=3$ with 10 explants per replication). Bars denoted by the same letter indicate no significant difference at $p<0.05$ according to Duncan's multiple range tests. Bar $=1 \mathrm{~cm}$. Photographs $\mathbf{( B )}$ show hypocotyl explants after 7 days of the treatments indicated.

ZnPPIX. Thus, CO might be involved in $\mathrm{H}_{2}$-induced adventitious root formation under drought condition.

\section{Effect of Pretreatments with $\mathrm{H}_{2}$ and $\mathrm{CO}$ on Leaf Relative Water Content during Adventitious Rooting under Simulated Drought Stress}

Leaf RWC decreased continuously with the progressive drought in all treatments (Figure 3). Compared with the control, PEG treatment resulted in an obvious reduction in RWC. The RWC in treatments with HRW, CO, and CO+HRW were significantly higher than that in PEG treatment at 24 and $48 \mathrm{~h}(P<0.05)$. Compared with PEG treatment, HRW, CO, and CO+HRW treatments increased RWC by $3.95,7.72$, and $5.12 \%$ at $48 \mathrm{~h}$, respectively. When $\mathrm{Hb}$ or ZnPPIX was added to $\mathrm{HRW} / \mathrm{CO}$, RWC significantly decreased at 24 and $48 \mathrm{~h}(P<0.05)$. These results indicate that PEG reduced RWC during adventitious rooting and $\mathrm{H}_{2}$ and $\mathrm{CO}$ alleviated the reduction. Meanwhile, $\mathrm{Hb}$ or ZnPPIX partially reversed the positive effects of $\mathrm{H}_{2}$ and $\mathrm{CO}$ on RWC. 


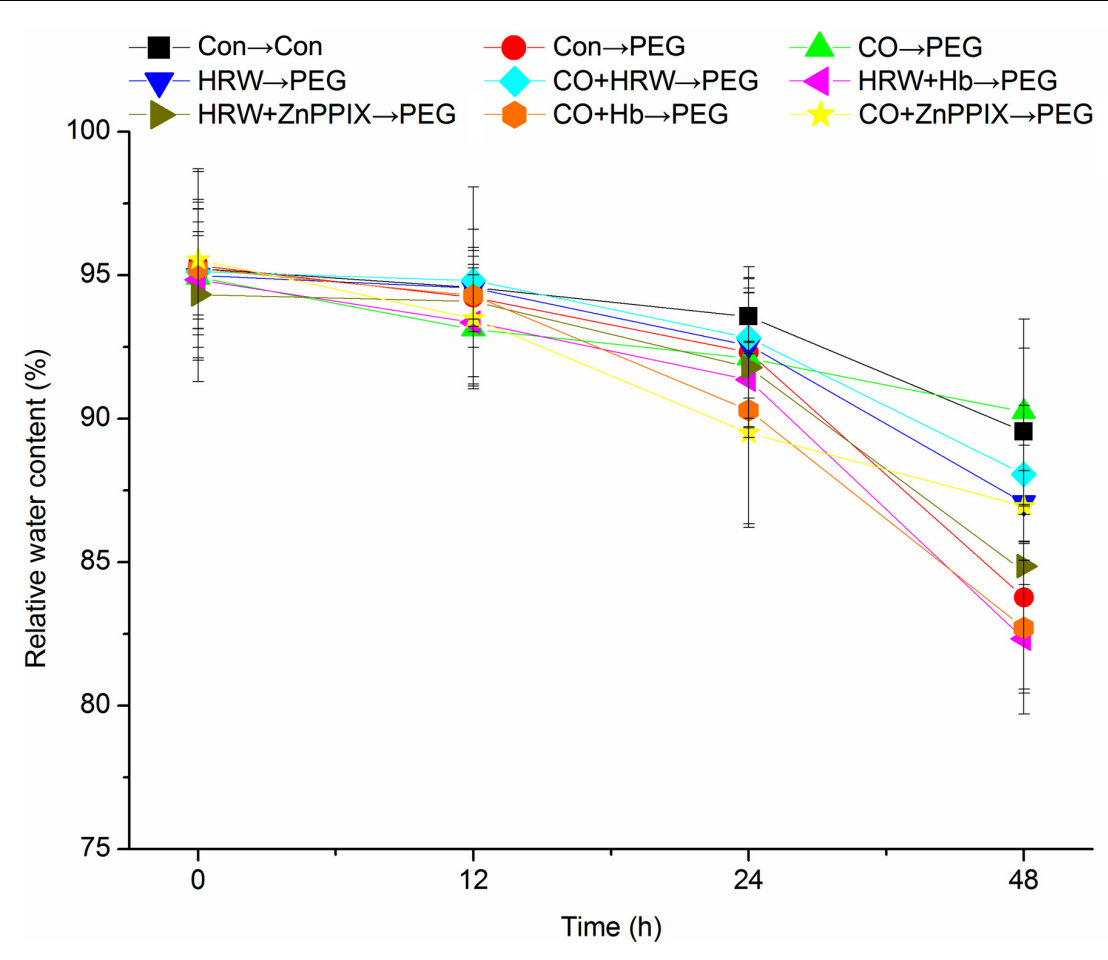

FIGURE 3 | Effects of $\mathbf{H}_{2}$ and $\mathbf{C O}$ on the relative water content in cucumber explants leaves under drought stress. Rooting were Con (square), PEG (circle), $\mathrm{CO}$ (regular triangle), HRW (inverted triangle), $\mathrm{CO}+\mathrm{HRE}$ (diamond), HRW+Hb (left triangle), HRW+ZnPPIX (right triangle), CO+Hb (hexagon), or CO+ZnPPIX (pentagram) as indicated. The concentrations of PEG, HRW, and CO were used at $0.3 \%(\mathrm{w} / \mathrm{v}), 50 \%$ ( $/ \mathrm{v})$, and $500 \mu \mathrm{M}$, respectively. Vertical bars represent mean \pm SE value from three independent experiments.

\section{Effect of Pretreatments with $\mathrm{H}_{2}$ and $\mathrm{CO}$ on Leaf Chlorophyll Content and Chlorophyll Fluorescence Parameters during Adventitious Rooting under Simulated Drought Stress}

Chlorophyll quantification has been widely used to estimate plant response to various stresses. Therefore, the effects of HRW and $\mathrm{CO}$ on leaf chlorophyll content were evaluated during adventitious rooting under drought condition. Compared with control, the contents of chl a and chl a+b decreased at 12, 24, and $48 \mathrm{~h}$ in PEG treatment explants (Table 1). Compared with PEG, the chl a and chl $\mathrm{a}+\mathrm{b}$ content in treatments with $\mathrm{HRW}, \mathrm{CO}$, and $\mathrm{CO}+\mathrm{HRW}$ significantly increased during $0-48 \mathrm{~h}$. When $\mathrm{Hb}$ and ZnPPIX were added to HRW and CO, the chl a and chl a+b content decreased at 0-48 h. Compared with the control, drought stress (PEG treatment) resulted in a decrease in chl b content at 12,24 , and $48 \mathrm{~h}$. However, a significant increase in the chl $\mathrm{b}$ content was observed in the treatments with $\mathrm{HRW}, \mathrm{CO}$ and $\mathrm{CO}+\mathrm{HRW}$ during $12-48 \mathrm{~h}$. A significant decrease in chl b in HRW/CO+Hb and HRW/CO+ZnPPIX treatments were observed at 12-48 h, compared with the HRW (Table 1). Therefore, $\mathrm{H}_{2}$ and $\mathrm{CO}$ may maintain chlorophyll content under drought stress during adventitious rooting, and $\mathrm{CO}$ might be involved in $\mathrm{H}_{2}$-induced chlorophyll content promotion in cucumber.
The effects of $\mathrm{H}_{2}$ and $\mathrm{CO}$ on chlorophyll fluorescence parameters were studied during adventitious rooting under drought stress. During the experiment period, there was a continued decrease for all chlorophyll fluorescence parameters in PEG, $\mathrm{HRW} / \mathrm{CO}+\mathrm{Hb}$ and $\mathrm{HRW} / \mathrm{CO}+\mathrm{ZnPPIX}$ treatments (Figure 4). These parameters in HRW treatment reached minimum at $24 \mathrm{~h}$ and then increased until the end. When the cucumber explants were transferred to PEG treatment, the $\mathrm{Fv} / \mathrm{Fm}$ decreased at 12,24 , and $48 \mathrm{~h}$. However, HRW, CO, and $\mathrm{CO}+\mathrm{HRW}$ treatments increased the $\mathrm{Fv} / \mathrm{Fm}$ at 12,24 , and $48 \mathrm{~h}$ compared with PEG treatment. Furthermore, when the $\mathrm{Hb}$ or ZnPPIX was added to HRW/CO, the positive effect of $\mathrm{HRW} / \mathrm{CO}$ on the parameters was significantly reversed $(P<0.05$; Figure 4A).

Compared with the control, PEG treatment significantly reduced the $\Phi P S I I$ at 12,24 , and $48 \mathrm{~h}$ (Figure $4 \mathrm{~B}$ ). In addition, treatments with $\mathrm{HRW}, \mathrm{CO}$, and $\mathrm{CO}+\mathrm{HRW}$ yielded significantly higher $\Phi$ PSII values than PEG treatment $(P<0.05)$. CO treatment yielded maximum values among all treatments during the period. Meanwhile, the explants treated with $\mathrm{HRW} / \mathrm{CO}+\mathrm{Hb}$ and HRW/CO+ZnPPIX showed lower $\Phi$ PSII than those treated with $\mathrm{HRW} / \mathrm{CO}$ (Figure 4B).

Figure 4C showed that the tendency of $\mathrm{qP}$ changed almost concurrently with that of $\mathrm{Fv} / \mathrm{Fm}$. It appeared that HRW, CO, and $\mathrm{CO}+\mathrm{HRW}$ could alleviate the negative effect of PEG on qP. However, the addition of $\mathrm{Hb}$ or ZnPPIX in HRW/CO 
TABLE 1 | Effect of $\mathrm{H}_{2}$ and $\mathrm{CO}$ on leaf chlorophyll content during adventitious rooting in cucumber explants under drought stress.

\begin{tabular}{|c|c|c|c|c|c|}
\hline \multirow[t]{2}{*}{ Index } & \multirow[t]{2}{*}{ Treatments } & \multicolumn{4}{|c|}{ Time (h) } \\
\hline & & 0 & 12 & 24 & 48 \\
\hline \multirow[t]{9}{*}{ Chl a } & Con $\rightarrow$ Con & $1.14 \pm 0.09 c$ & $1.11 \pm 0.02 a$ & $1.16 \pm 0.04 a$ & $1.16 \pm 0.11 a$ \\
\hline & Con $\rightarrow$ PEG & $1.14 \pm 0.03 c$ & $0.91 \pm 0.10 c$ & $0.79 \pm 0.11 c$ & $0.41 \pm 0.03 d$ \\
\hline & $\mathrm{HRW} \rightarrow \mathrm{PEG}$ & $1.18 \pm 0.03 b$ & $1.09 \pm 0.03 b$ & $0.93 \pm 0.04 b$ & $0.93 \pm 0.03 b$ \\
\hline & $\mathrm{CO} \rightarrow \mathrm{PEG}$ & $1.26 \pm 0.04 a$ & $1.11 \pm 0.02 a$ & $1.16 \pm 0.03 a$ & $1.19 \pm 0.03 a$ \\
\hline & $\mathrm{CO}+\mathrm{HRW} \rightarrow \mathrm{PEG}$ & $1.16 \pm 0.05 a$ & $1.13 \pm 0.02 a$ & $1.14 \pm 0.01 a$ & $1.21 \pm 0.08 a$ \\
\hline & $\mathrm{HRW}+\mathrm{Hb} \rightarrow \mathrm{PEG}$ & $1.09 \pm 0.02 d$ & $0.85 \pm 0.02 d$ & $0.75 \pm 0.09 d$ & $0.38 \pm 0.02 d$ \\
\hline & $\mathrm{HRW}+\mathrm{ZnPPIX} \rightarrow \mathrm{PEG}$ & $1.11 \pm 0.03 d$ & $0.87 \pm 0.10 \mathrm{~cd}$ & $0.76 \pm 0.09 \mathrm{~cd}$ & $0.39 \pm 0.02 d$ \\
\hline & $\mathrm{CO}+\mathrm{Hb} \rightarrow \mathrm{PEG}$ & $1.15 \pm 0.08 c$ & $0.84 \pm 0.04 \mathrm{e}$ & $0.79 \pm 0.02 c$ & $0.43 \pm 0.05 \mathrm{~cd}$ \\
\hline & $\mathrm{CO}+\mathrm{ZnPPIX} \rightarrow \mathrm{PEG}$ & $1.15 \pm 0.05 c$ & $0.86 \pm 0.15 d$ & $0.77 \pm 0.06 \mathrm{~cd}$ & $0.46 \pm 0.07 c$ \\
\hline \multirow[t]{9}{*}{ Chl b } & Con $\rightarrow$ Con & $0.48 \pm 0.01 a$ & $0.47 \pm 0.04 a b$ & $0.47 \pm 0.03 a b$ & $0.45 \pm 0.03 a b$ \\
\hline & Con $\rightarrow$ PEG & $0.47 \pm 0.02 a$ & $0.30 \pm 0.03 b$ & $0.25 \pm 0.02 c$ & $0.13 \pm 0.02 c$ \\
\hline & $\mathrm{HRW} \rightarrow \mathrm{PEG}$ & $0.48 \pm 0.01 a$ & $0.44 \pm 0.04 a$ & $0.37 \pm 0.02 b$ & $0.34 \pm 0.03 b$ \\
\hline & $\mathrm{CO} \rightarrow \mathrm{PEG}$ & $0.49 \pm 0.03 a$ & $0.47 \pm 0.03 a b$ & $0.47 \pm 0.01 a b$ & $0.45 \pm 0.02 a$ \\
\hline & $\mathrm{CO}+\mathrm{HRW} \rightarrow \mathrm{PEG}$ & $0.51 \pm 0.05 a$ & $0.49 \pm 0.02 a$ & $0.48 \pm 0.04 a$ & $0.46 \pm 0.03 a$ \\
\hline & $\mathrm{HRW}+\mathrm{Hb} \rightarrow \mathrm{PEG}$ & $0.47 \pm 0.02 a$ & $0.29 \pm 0.02 c$ & $0.25 \pm 0.01 c$ & $0.12 \pm 0.02 c$ \\
\hline & $\mathrm{HRW}+\mathrm{ZnPPIX} \rightarrow \mathrm{PEG}$ & $0.47 \pm 0.05 a$ & $0.30 \pm 0.05 b$ & $0.24 \pm 0.01 d$ & $0.12 \pm 0.02 c$ \\
\hline & $\mathrm{CO}+\mathrm{Hb} \rightarrow \mathrm{PEG}$ & $0.46 \pm 0.02 a$ & $0.30 \pm 0.05 b$ & $0.25 \pm 0.05 c$ & $0.12 \pm 0.05 c$ \\
\hline & $\mathrm{CO}+\mathrm{ZnPPIX} \rightarrow \mathrm{PEG}$ & $0.47 \pm 0.04 a$ & $0.31 \pm 0.03 b$ & $0.25 \pm 0.03 c$ & $0.11 \pm 0.06 d$ \\
\hline \multirow[t]{9}{*}{ Chla+b } & Con $\rightarrow$ Con & $1.62 \pm 0.10 \mathrm{bc}$ & $1.58 \pm 0.03 a$ & $1.63 \pm 0.07 a$ & $1.61 \pm 0.10 a$ \\
\hline & Con $\rightarrow$ PEG & $1.61 \pm 0.04 b c$ & $1.21 \pm 0.09 b$ & $1.04 \pm 0.11 c$ & $0.54 \pm 0.02 c$ \\
\hline & $\mathrm{HRW} \rightarrow \mathrm{PEG}$ & $1.66 \pm 0.02 b$ & $1.53 \pm 0.01 a$ & $1.31 \pm 0.03 b$ & $1.28 \pm 0.06 b$ \\
\hline & $\mathrm{CO} \rightarrow \mathrm{PEG}$ & $1.75 \pm 0.04 a$ & $1.58 \pm 0.01 a$ & $1.64 \pm 0.02 a$ & $1.64 \pm 0.03 a$ \\
\hline & $\mathrm{CO}+\mathrm{HRW} \rightarrow \mathrm{PEG}$ & $1.81 \pm 0.01 \mathrm{a}$ & $1.59 \pm 0.03 a$ & $1.63 \pm 0.04 a$ & $1.61 \pm 0.05 a$ \\
\hline & $\mathrm{HRW}+\mathrm{Hb} \rightarrow \mathrm{PEG}$ & $1.55 \pm 0.04 c$ & $1.14 \pm 0.04 d$ & $0.99 \pm 0.01 d$ & $0.50 \pm 0.03 c$ \\
\hline & $\mathrm{HRW}+\mathrm{ZnPPIX} \rightarrow \mathrm{PEG}$ & $1.59 \pm 0.04 \mathrm{bc}$ & $1.17 \pm 0.10 \mathrm{cb}$ & $1.00 \pm 0.10 c$ & $0.51 \pm 0.04 c$ \\
\hline & $\mathrm{CO}+\mathrm{Hb} \rightarrow \mathrm{PEG}$ & $1.55 \pm 0.05 c$ & $1.19 \pm 0.02 c$ & $1.01 \pm 0.03 c$ & $0.50 \pm 0.05 c$ \\
\hline & $\mathrm{CO}+\mathrm{ZnPPIX} \rightarrow \mathrm{PEG}$ & $1.58 \pm 0.02 b c$ & $1.21 \pm 0.05 b$ & $0.99 \pm 0.01 d$ & $0.50 \pm 0.03 c$ \\
\hline
\end{tabular}

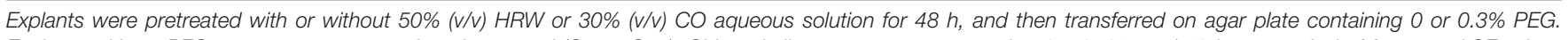

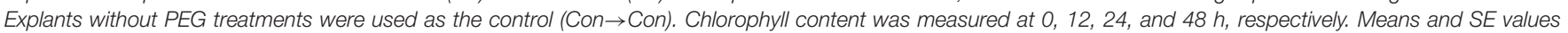

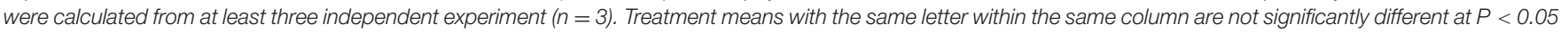
according to Duncan's multiple range tests.

suppressed the stimulating effect of $\mathrm{HRW} / \mathrm{CO}$ on the $\mathrm{qP}$ under drought condition. For example, the $\mathrm{qP}$ in $\mathrm{HRW}+\mathrm{Hb}$ and HRW+ZnPPIX treatments at $48 \mathrm{~h}$ were 19.46 and $17.54 \%$ lower than that in HRW treatment, respectively; the $\mathrm{qP}$ in $\mathrm{CO}+\mathrm{Hb}$ and $\mathrm{CO}+\mathrm{ZnPPIX}$ treatments at $48 \mathrm{~h}$ were 9.85 and $13.23 \%$ lower than that in $\mathrm{CO}$ treatment, respectively. These results suggest that both $\mathrm{H}_{2}$ and $\mathrm{CO}$ affected chlorophyll fluorescence parameters during adventitious rooting under drought stress.

\section{Effect of Pretreatments with $\mathrm{H}_{2}$ and $\mathrm{CO}$ on the Levels of TBARS, $\mathrm{O}_{2}{ }^{-}$, and $\mathrm{H}_{2} \mathrm{O}_{2}$ during Adventitious Rooting under Simulated Drought Stress}

As shown in Figure 5, the levels of TBARS, $\mathrm{O}_{2}{ }^{-}$, and $\mathrm{H}_{2} \mathrm{O}_{2}$ in the control explants remained constant during the period. The levels of TBARS, $\mathrm{O}_{2}{ }^{-}$, and $\mathrm{H}_{2} \mathrm{O}_{2}$ in PEG, $\mathrm{HRW} / \mathrm{CO}+\mathrm{Hb}$, and $\mathrm{HRW} / \mathrm{CO}+\mathrm{ZnPPIX}$ treatments continuously increased to the end of the experiment. In addition, Figure 5A showed that the TBARS level in HRW, CO, and CO+HRW treatments reached the highest at $24 \mathrm{~h}$ and then decreased. Whereas the levels of $\mathrm{O}_{2}{ }^{-}$and $\mathrm{H}_{2} \mathrm{O}_{2}$ in $\mathrm{HRW}, \mathrm{CO}$, and $\mathrm{CO}+\mathrm{HRW}$ treatments increased during $0-12 \mathrm{~h}$, and then decreased after $12 \mathrm{~h}$ (Figures 5B,C). Compared with the control, PEG treatment caused $162.77,320.94$, and $592.38 \%$ increase in TBARS level at 12, 24, and $48 \mathrm{~h}$, respectively (Figure 5A). When explants were transferred to PEG treatment, HRW-, CO-, and CO+HRWpretreated explants had reduced TBARS levels at 12, 24, and $48 \mathrm{~h}$ compared with PEG treatment. However, HRW/CO+Hband HRW/CO+ZnPPIX-pretreated explants had higher TBARS levels than only HRW/CO- and $\mathrm{CO}+\mathrm{HRW}$-pretreated explants at 12, 24, and $48 \mathrm{~h}$ (Figure 5A).

PEG treatment resulted in significant higher levels of $\mathrm{O}_{2}{ }^{-}$and $\mathrm{H}_{2} \mathrm{O}_{2}$ than those in the control at 12, 24, and $48 \mathrm{~h}$ (Figures 5B,C). Meanwhile, $\mathrm{HRW}, \mathrm{CO}$, and $\mathrm{CO}+\mathrm{HRW}$ treatments significantly decreased the levels of $\mathrm{O}_{2}{ }^{-}$and $\mathrm{H}_{2} \mathrm{O}_{2}$ in cucumber explants, compared with PEG treatment. When $\mathrm{Hb}$ and ZnPPIX were added into $\mathrm{HRW} / \mathrm{CO}$ treatment, the effects of $\mathrm{HRW} / \mathrm{CO}$ on the levels of $\mathrm{O}_{2}{ }^{-}$and $\mathrm{H}_{2} \mathrm{O}_{2}$ were reversed (Figures $5 \mathrm{~B}, \mathrm{C}$ ). Therefore, $\mathrm{CO}$ might be involved in $\mathrm{H}_{2}$-adjusted TBARS, $\mathrm{O}_{2}{ }^{-}$, and $\mathrm{H}_{2} \mathrm{O}_{2}$ content during adventitious rooting under drought stress. 


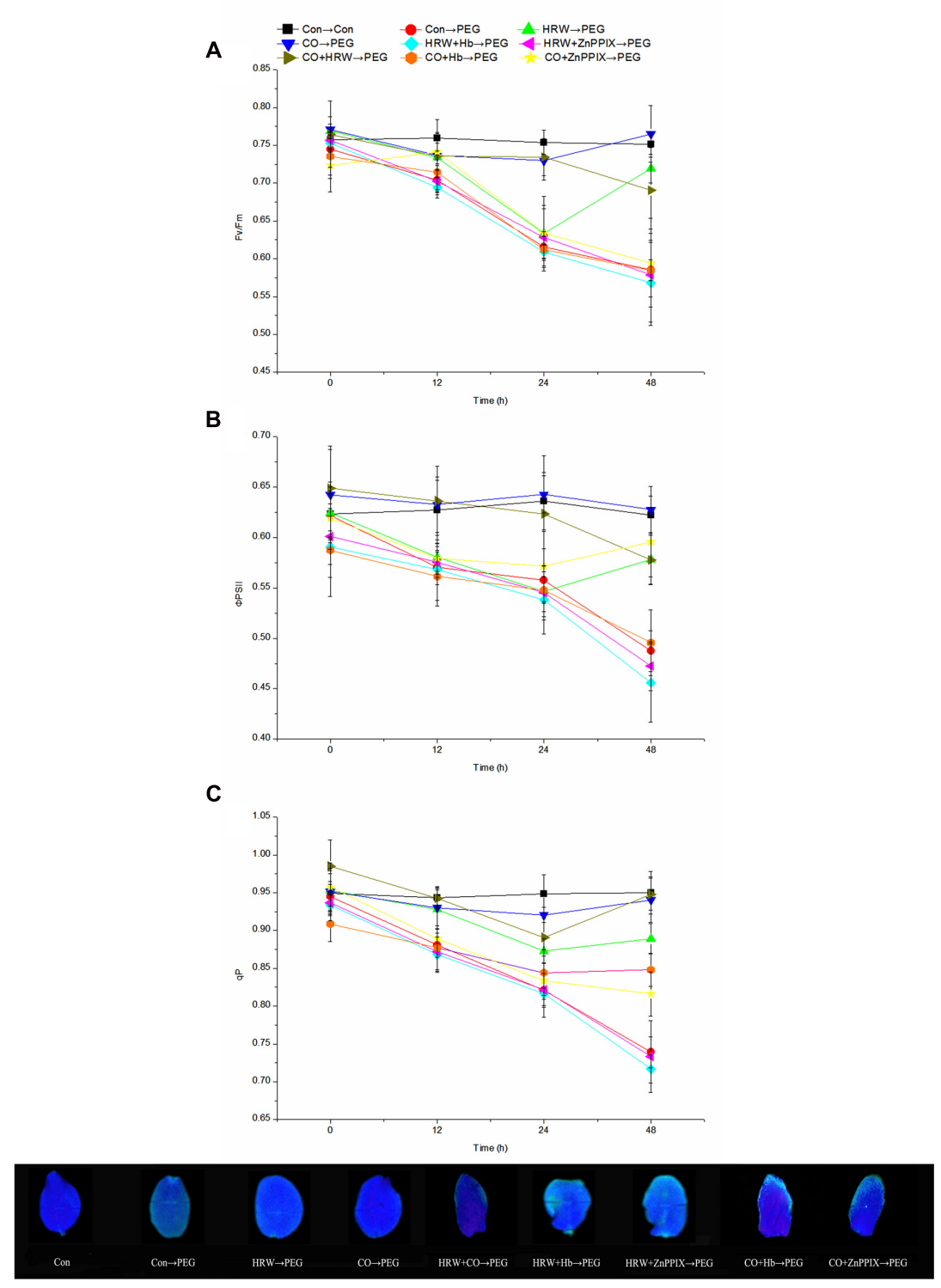

FIGURE 4 | Effects of $\mathrm{H}_{2}$ and $\mathrm{CO}$ on chlorophyll fluorescence parameters in the cotyledon of cucumber explant during the adventitious rooting process. Rooting were Con (square), PEG (circle), CO (regular triangle), HRW (inverted triangle), CO+HRW (diamond), HRW+Hb (left triangle), HRW+ZnPPIX (right triangle), $\mathrm{CO}+\mathrm{Hb}$ (hexagon), or $\mathrm{CO}+\mathrm{ZnPPIX}$ (pentagram) as indicated. The concentrations of PEG, HRW, and CO were used at $0.3 \%$ (w/v), $50 \%$ (v/v), and $500 \mu \mathrm{M}$, respectively. Fv/Fm (A), ФPS II (B), and qP (C) were determined after 0, 12, 24, 48 h of PEG treatment. Vertical bars represent mean \pm SE value from three independent experiments.

\section{Effect of Pretreatments with $\mathrm{H}_{2}$ and $\mathrm{CO}$ on Activities of Antioxidant Enzymes during Rooting under Simulated Drought Stress}

As shown in Figures 6A,B, the activities of SOD and POD in the control treatment remained constant throughout the $48 \mathrm{~h}$ period of the experiment. Under drought condition, these activities in HRW-, CO-, and $\mathrm{CO}+\mathrm{HRW}$-pretreated explants rapidly increased at $0-24 \mathrm{~h}$, followed by a gradual decrease until $48 \mathrm{~h}$. The activities of SOD and POD in PEG treatment were slightly decreased within 12-48 h, whereas they significantly decreased in $\mathrm{HRW} / \mathrm{CO}+\mathrm{Hb}$ - and $\mathrm{HRW} / \mathrm{CO}+\mathrm{ZnPPIX}$-pretreated explants during $0-48 \mathrm{~h}(P<0.05)$. The activities of SOD and POD in $\mathrm{PEG}$ treatment were significantly lower than those in the control $(P<0.05$; Figures 6A,B). The activities of SOD and POD in explants pretreated with HRW, CO, and CO+HRW were significantly higher than those in explants treated with PEG $(P<0.05)$. For example, POD activity in HRW treatment was 2.86-, 3.09-, and 3.27-fold increases over those in PEG treatment at 12,24 , and $48 \mathrm{~h}$, respectively. Meanwhile, the activities of SOD and POD in $\mathrm{HRW} / \mathrm{CO}+\mathrm{Hb}$ - or $\mathrm{HRW} / \mathrm{CO}+\mathrm{ZnPPIX}$-pretreated 

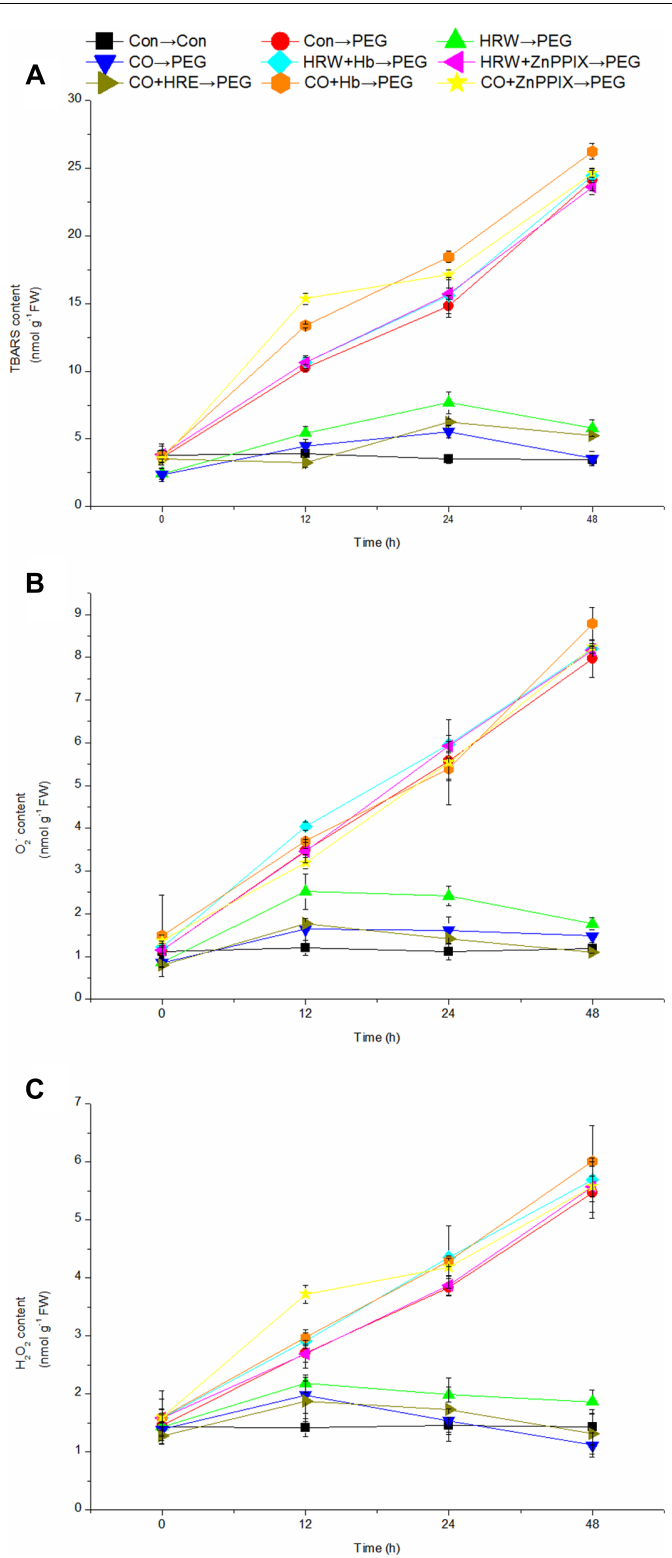

FIGURE 5 | Effects of $\mathrm{H}_{2}$ and $\mathrm{CO}$ on the levels of TBARS, $\mathrm{O}_{2}{ }^{-}$, and $\mathrm{H}_{2} \mathrm{O}_{2}$ in the adventitious root of cucumber during the rooting process. Rooting were Con (square), PEG (circle), CO (regular triangle), HRW (inverted triangle), $\mathrm{CO}+\mathrm{HRW}$ (diamond), HRW+Hb (left triangle), HRW+ZnPPIX (right triangle), $\mathrm{CO}+\mathrm{Hb}$ (hexagon), or $\mathrm{CO}+\mathrm{ZnPPIX}$ (pentagram) as indicated. The concentrations of PEG, HRW, and CO were used at $0.3 \%(\mathrm{w} / \mathrm{v}), 50 \%(\mathrm{v} / \mathrm{v})$, and $500 \mu \mathrm{M}$, respectively. The content of TBARS (A), $\mathrm{O}_{2}{ }^{-}$(B), and $\mathrm{H}_{2} \mathrm{O}_{2}$ (C) was determined after $0,12,24,48 \mathrm{~h}$ of PEG treatment. Vertical bars represent mean \pm SE value from three independent experiments.

explants were lower than those in HRW/CO- pretreated explants (Figures 6A,B).

The CAT activity remained constant in the control within 0-48 h (Figure 6C). Under drought stress, the CAT activity of explants pretreated with HRW decreased during $0-12 \mathrm{~h}$, and then increased sharply at $12-48 \mathrm{~h}$. However, the CAT activity in explants pretreated with $\mathrm{CO}, \mathrm{HRW}$, and $\mathrm{CO}+\mathrm{HRW}$ markedly increased during $0-48 \mathrm{~h}(P<0.05)$. The CAT activity in explants pretreated with PEG, $\mathrm{HRW} / \mathrm{CO}+\mathrm{Hb}$, and $\mathrm{HRW} / \mathrm{CO}+\mathrm{ZnPPIX}$ was slightly increased from 12 to $24 \mathrm{~h}$ and then decreased until $48 \mathrm{~h}$. The CAT activity of in PEG treatment was 22.03, 16.31, and $28.45 \%$ lower than that in the control at 12,24 , and $48 \mathrm{~h}$, respectively. The CAT activity in explants pretreated with $\mathrm{HRW}, \mathrm{CO}$, and $\mathrm{CO}+\mathrm{HRW}$ was significantly higher than that in explants treated with PEG $(P<0.05)$. The CAT activity in the HRW $+\mathrm{Hb}, \mathrm{HRW}+\mathrm{ZnPPIX}, \mathrm{CO}+\mathrm{Hb}$, and $\mathrm{CO}+\mathrm{ZnPPIX}$ treatments was $72.12,71.24,78.66$, and $74.35 \%$ lower than that in HRW treatment at $48 \mathrm{~h}$, respectively (Figure $6 \mathrm{C}$ ).

As shown in Figure 6D, the APX activity in control treatment remained constant within $0-48 \mathrm{~h}$. When explants were transferred to PEG treatment, the activity increased within $0-24 \mathrm{~h}$ and then slightly decreased within $24-48 \mathrm{~h}$ in HRW, $\mathrm{CO}$, and $\mathrm{CO}+\mathrm{HRW}$ pretreatments. In PEG, $\mathrm{HRW} / \mathrm{CO}+\mathrm{Hb}$ and HRW/CO+ZnPPIX treatments, the APX activity continuously decreased within $0-48 \mathrm{~h}$. The activity of APX in PEG treatment was 6.06 and $50.00 \%$ lower than that in the control at 24 and $48 \mathrm{~h}$, respectively. $\mathrm{HRW}, \mathrm{CO}$, and $\mathrm{CO}+\mathrm{HRW}$ treatments resulted in significantly higher APX activity than PEG treatment $(P<0.05)$. For example, when compared with PEG treatment, the APX activity in HRW, CO, and CO+HRW treatments increased by $303.22,819.35$, and $519.72 \%$ at $24 \mathrm{~h}$, respectively. The APX activity in HRW/CO+Hb or HRW/CO+ZnPPIX treatments was significantly lower than that in HRW treatment $\left(P<0.05\right.$; Figure 6D). Therefore, $\mathrm{CO}$ was involved in $\mathrm{H}_{2}-$ induced enhancement of the SOD, POD, CAT, and APX activities during adventitious rooting under drought condition.

\section{Effects of Pretreatments with $\mathrm{H}_{2}$ and $\mathrm{CO}$ on Levels of Osmotic Regulators during Adventitious Rooting under Simulated Drought Stress}

As showed in Figure 7A, the level of water soluble carbohydrate in the control slightly increased within $12-24 \mathrm{~h}$, and then decreased within 24-48 h. Under drought stress, the water soluble carbohydrate content increased continuously in HRW, $\mathrm{CO}$, and $\mathrm{CO}+\mathrm{HRW}$ treatments during $0-48 \mathrm{~h}$. However, it decreased in HRW/CO+Hb, HRW/CO+ZnPPIX, and PEG treatments from 12 to $48 \mathrm{~h}$. In addition, HRW, CO, and $\mathrm{CO}+\mathrm{HRW}$ treatments caused a significant increase in water soluble carbohydrate content compare with PEG treatment $(P<0.05)$. Simultaneously, $\mathrm{HRW} / \mathrm{CO}+\mathrm{Hb}$ and $\mathrm{HRW} / \mathrm{CO}+\mathrm{ZnPPIX}$ treatments suppressed the positive effect of $\mathrm{HRW} / \mathrm{CO}$ treatments. The water soluble carbohydrate content in the HRW $+\mathrm{Hb}, \mathrm{HRW}+\mathrm{ZnPPIX}, \mathrm{CO}+\mathrm{Hb}$, and $\mathrm{CO}+\mathrm{ZnPPIX}$ treatments was $68.87,68.77,71.42$, and $68.39 \%$ lower than that in HRW treatment at $48 \mathrm{~h}$, respectively (Figure 7A).

As shown in Figure 7B, the soluble protein content in the control decreased during 12-24 h, and then increased until $48 \mathrm{~h}$. Under drought condition, the soluble protein content increased continuously in HRW and CO pretreatments during $12-48 \mathrm{~h}$. The soluble protein content in CO+HRW treatment increased during $0-24 \mathrm{~h}$, and then decreased until $48 \mathrm{~h}$. In addition, the soluble protein content showed continued decrease 


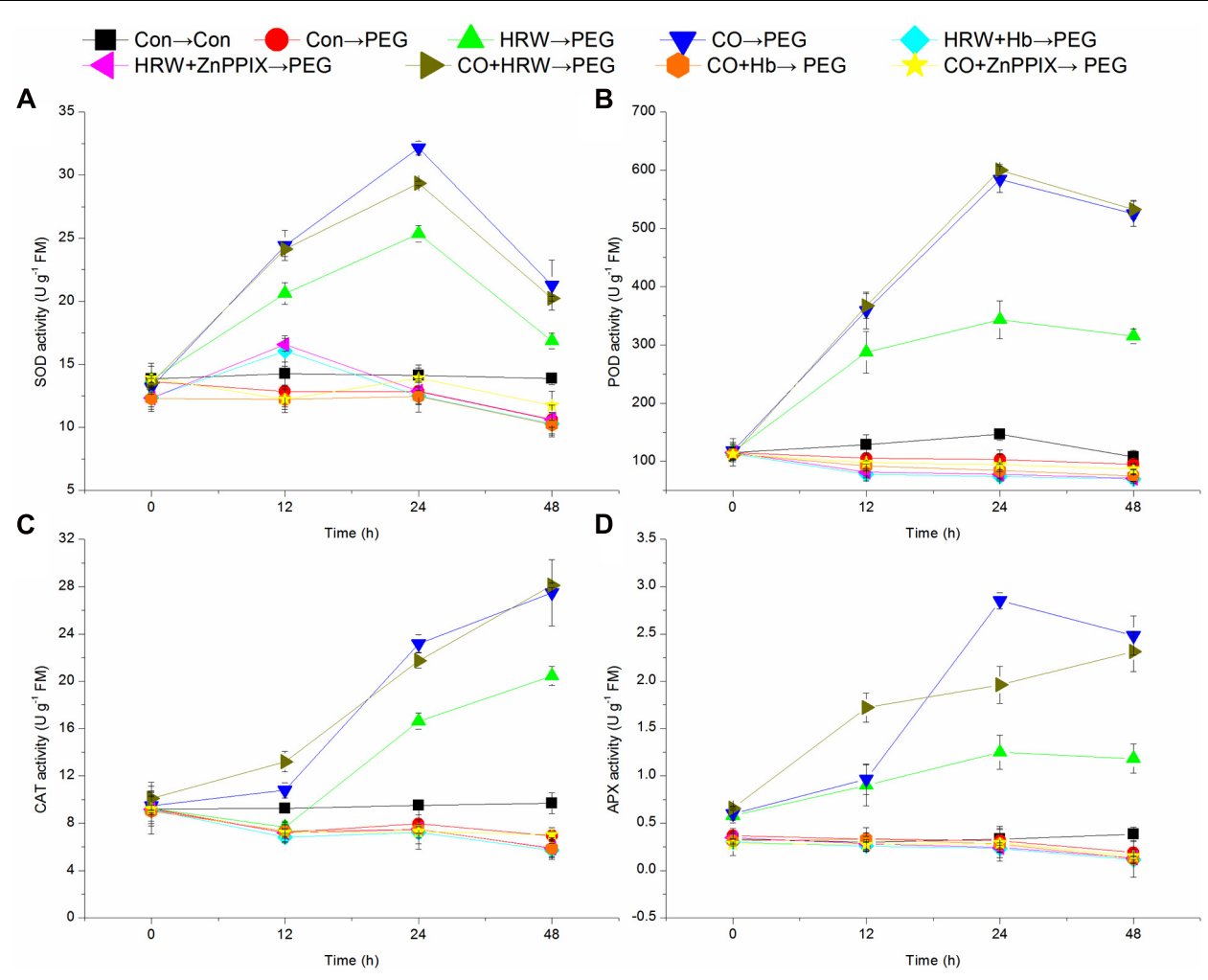

FIGURE 6 | Changes of antioxidant enzymes in cucumber explants during the rooting process. Rooting were Con (square), PEG (circle), CO (regular triangle), $\mathrm{HRW}$ (inverted triangle), $\mathrm{CO}+\mathrm{HRW}$ (diamond), HRW+Hb (left triangle), HRW+ZnPPIX (right triangle), $\mathrm{CO}+\mathrm{Hb}$ (hexagon), or CO+ZnPPIX (pentagram) as indicated. The concentrations of PEG, HRW, and CO were used at 0.3\% (w/v), $50 \%$ (v/v), and $500 \mu \mathrm{M}$, respectively. The activity of SOD (A), POD (B), CAT (C), and APX (D) was determined after 0, 12, 24, $48 \mathrm{~h}$ of PEG treatment. Vertical bars represent mean \pm SE value from three independent experiments.

in $\mathrm{HRW} / \mathrm{CO}+\mathrm{Hb}$, HRW/CO+ZnPPIX, and PEG treatments during $0-48$ h. Compared with the control, PEG treatment caused $31.85,43.76$, and $76.96 \%$ decrease in soluble protein content at 12, 24, and $48 \mathrm{~h}$, respectively. Compared with the PEG treatment, $\mathrm{HRW}, \mathrm{CO}$, and $\mathrm{CO}+\mathrm{HRW}$ treatments resulted in significantly higher soluble protein content $(P<0.05)$. However, if $\mathrm{Hb}$ or $\mathrm{ZnPPIX}$ were added to $\mathrm{HRW} / \mathrm{CO}$, the positive effect of $\mathrm{HRW} / \mathrm{CO}$ was reversed (Figure 7B).

The proline content remains constant during the study period (Figure 7C). Its content increased continuously in $\mathrm{HRW}, \mathrm{CO}$, and $\mathrm{CO}+\mathrm{HRW}$ treatments during 0-48 h. In addition, the proline content in PEG, $\mathrm{HRW} / \mathrm{CO}+\mathrm{Hb}$, and HRW+ZnPPIX treatments increased during 0-24 h, and then decreased during 24-48 h. The proline content continuously increased during 0-48 h in CO+ZnPPIX treatment. Compared with the control, a higher content of proline in PEG treatment was obtained. As showed in Figure 7C, pretreatments with $\mathrm{HRW}, \mathrm{CO}$ and $\mathrm{CO}+\mathrm{HRW}$ produced a significant increase in proline content compared with PEG treatment $(P<0.05)$. Compared with PEG treatment, $\mathrm{HRW}, \mathrm{CO}$, and $\mathrm{CO}+\mathrm{HRW}$ treatments resulted in 2.53-, 3.32-, and 3.19-fold proline content at $48 \mathrm{~h}$, respectively. When $\mathrm{Hb}$ or $\mathrm{ZnPPIX}$ were added to $\mathrm{HRW} / \mathrm{CO}$, it resulted in a significant reduction in proline content (Figure 7C). Therefore, $\mathrm{CO}$ could be involved in $\mathrm{H}_{2}$-induced accumulation of water soluble carbohydrate, soluble protein, and proline during adventitious rooting in cucumber under drought stress.

\section{DISCUSSION}

Drought stress is one of the main abiotic stresses that affects plant growth and development. Drought stress affects growth, water and nutrient relations, photosynthesis, assimilate partitioning, and respiration in plants (Farooq et al., 2009). Thus, studying the mechanism of plant drought tolerance can provide a theoretical basis for improved crop production in the future.

Carbon monoxide was regarded as a cell protection mechanism against abiotic-induced damage (Yannarelli et al., 2006; Liu et al., 2010; Zhang et al., 2012). For example, Zheng et al. (2011) also demonstrated that CO could alleviate the oxidative damage effects of heavy metals on plants. In the present study, our results indicated that the applications of both low concentration of the CO donor Hemin (10, 100, and $500 \mu \mathrm{M}, \mathrm{w} / \mathrm{v}$ ) and $\mathrm{CO}$ aqueous solution (10, 30, and $50 \%$, $\mathrm{v} / \mathrm{v}$ ) promoted adventitious rooting in cucumber explants under drought stress. However, high concentration of Hemin (1000 $\mu \mathrm{M}, \mathrm{w} / \mathrm{v}$ ) and CO aqueous solution (50 and 100\%, $\mathrm{v} / \mathrm{v}$ ) inhibited adventitious rooting under drought condition 

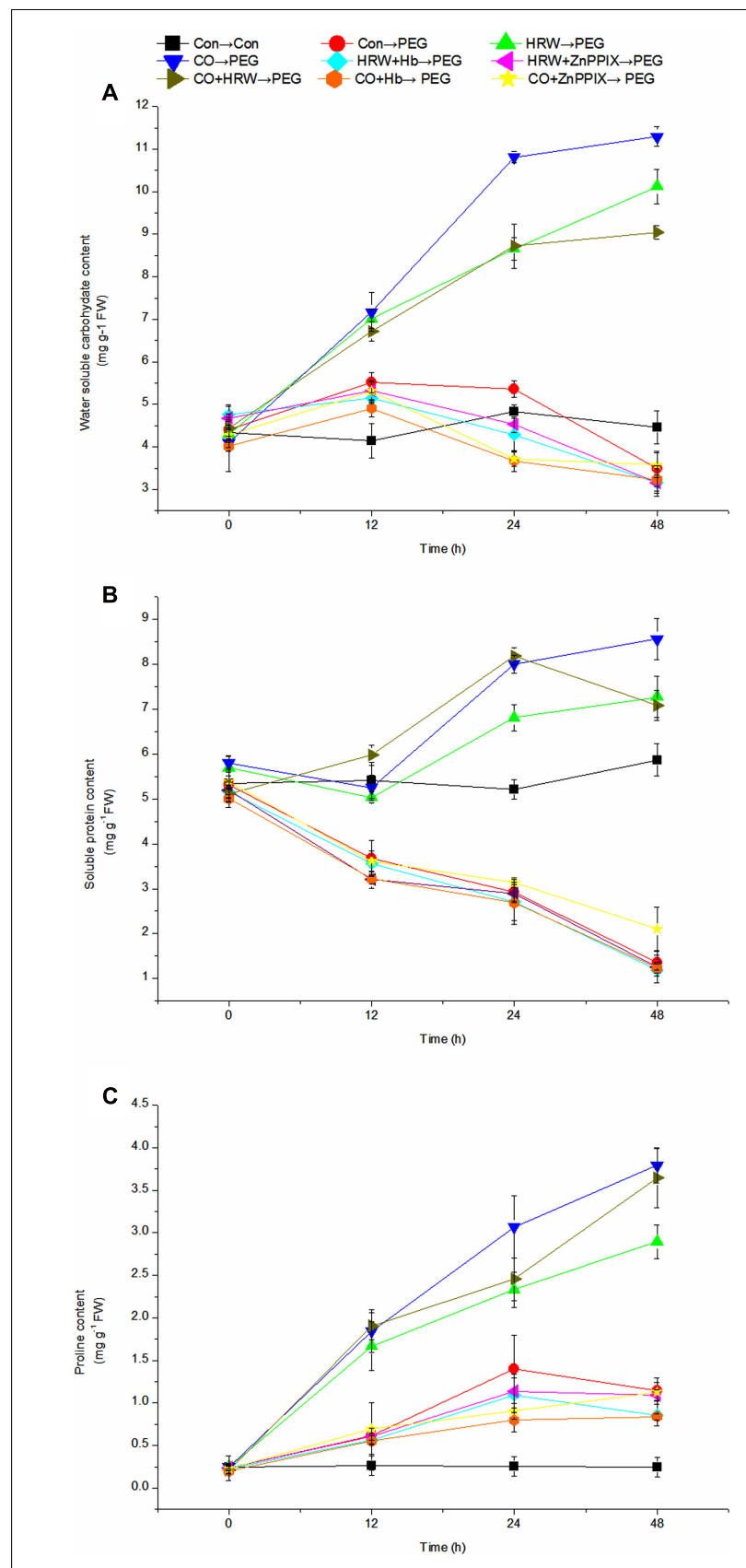

FIGURE 7 | Changes of osmotic regulator levels in cucumber explants during the rooting process. Rooting were Con (square), PEG (circle), CO (regular triangle), HRW (inverted triangle), $\mathrm{CO}+\mathrm{HRW}$ (diamond), $\mathrm{HRW}+\mathrm{Hb}$ (left triangle), $\mathrm{HRW}+\mathrm{ZnPPIX}$ (right triangle), $\mathrm{CO}+\mathrm{Hb}$ (hexagon), or $\mathrm{CO}+\mathrm{ZnPPIX}$ (pentagram) as indicated. The concentrations of PEG, HRW, and $\mathrm{CO}$ were used at $0.3 \%(\mathrm{w} / \mathrm{V}), 50 \%(\mathrm{v} / \mathrm{V})$, and $500 \mu \mathrm{M}$, respectively. The content of water soluble carbohydrate (A), soluble protein (B), and proline (C) was determined after $0,12,24,48 \mathrm{~h}$ of PEG treatment. Vertical bars represent mean \pm SE value from three independent experiments.

(Figure 1). The results are consistent with the findings of Dekker and Hargrove (2002), which showed that low concentration of CO $(0.1 \%)$ in the air stimulated seed germination, whereas high concentration (75\%) inhibited germination. Previous studies showed that $\mathrm{CO}$ exhibited positive effects on regulating plant root development in cucumber (Xuan et al., 2008). Xu et al. (2006) reported that the application of CO to mung bean hypocotyl induced de novo adventitious root formation in doseand time-dependent manners. Our results indicate that $\mathrm{CO}$ was not only involved in drought stress responses but also required for adventitious root development in cucumber. Thus, CO could alleviate various abiotics tresses during the plant adventitious rooting.

$\mathrm{H}_{2}$ had an obvious promotion effect on adventitious rooting under drought stress. Some results from previous research by others indicated that $\mathrm{H}_{2}$ had great potential applications within plant growth (Jin et al., 2013; Cui et al., 2014). $\mathrm{H}_{2}$ was found to enhance salt tolerance in Arabidopsis (Xie et al., 2012) and drought tolerance in Arabidopsis (Xie et al., 2014) and in M. sativa (Jin et al., 2016). Recent studies revealed that $\mathrm{H}_{2}$ was involved in alleviating ultraviolet $\mathrm{A}$ and ultraviolet $\mathrm{B}$ irradiation in two varieties of radish sprouts and in M. sativa (Su et al., 2014; Xie et al., 2015). Our results were consistent with the results of $\mathrm{Wu}$ et al. (2015), who found that $\mathrm{H}_{2}$ promoted root growth in Chinese cabbage under Cd stress. Xie et al. (2012) reported that a high concentration of HRW had a negative effect on the primary root growth in Arabidopsis seedlings under salinity stress. These findings are indications that suitable concentration of $\mathrm{H}_{2}$ has important functional implications in promoting growth and development of plants under abiotic stresses.

Studies have shown that $\mathrm{H}_{2}$ signal transduction pathways do not always work dependently. Zhu et al. (2016b) demonstrated that $\mathrm{NO}$ is required for $\mathrm{H}_{2}$-induced adventitious rooting in cucumber. CO alleviated osmotic-induced wheat seed germination inhibition and lipid peroxidation which required the participation of NO (Liu et al., 2010). Furthermore, $\mathrm{H}_{2}$ was involved in phytohormone and stress responses in plants (Zeng et al., 2013). A functional interaction of CO and phytohormone has been demonstrated in regulating plant growth and development (Wang and Liao, 2016). Jin et al. (2013) found that $\mathrm{H}_{2} \mathrm{O}_{2}$ and heme oxygenase- 1 were involved in $\mathrm{H}_{2}$-induced osmotic stress tolerance in alfalfa. She and Song (2008) illustrated that CO-induced stomatal closure probably was mediated by $\mathrm{H}_{2} \mathrm{O}_{2}$ signaling pathways in $\mathrm{V}$. Faba. These results indicated that both the crosstalk among $\mathrm{H}_{2}, \mathrm{CO}$, and other signal molecular optimized plants growth and enhanced the tolerance to various abiotic stresses. In addition, previous reports have shown that $\mathrm{H}_{2}$ may act synergistically with $\mathrm{CO}$ to enhance adventitious root development in non-stress conditions (Lin et al., 2014). In our study, we found that HRW, CO, and CO+HRW induced adventitious rooting under drought stress. Previous research demonstrated the cucumber produced CO at levels of 10 to $25 \mathrm{~mL}$ $\mathrm{L}^{-1}$ (Siegel et al., 1962). As a pharmacology method, ZnPPIX as a specific inhibitor of $\mathrm{CO}$ and $\mathrm{Hb}$ as $\mathrm{CO}$ scavenger were used to inhibit endogenous $\mathrm{CO}$ in $\mathrm{H}_{2} / \mathrm{CO}$-treated explants. Interestingly, the results showed that the addition of $\mathrm{Hb}$ or ZnPPIX into $\mathrm{H}_{2} / \mathrm{CO}$ significantly reversed the positive effect of $\mathrm{H}_{2} / \mathrm{CO}$. These results proved that $\mathrm{CO}$ was involved in $\mathrm{H}_{2}$-induced adventitious rooting under drought stress.

Previous study suggested that PEG treatment might reduce oxygen availability in plants (Chazen et al., 1995). In the study, 
PEG was used to simulate drought stress. Our results showed that PEG treatment significantly decreased leaf RWC during adventitious rooting, indicating that $\mathrm{PEG}$ treatment resulted in simulated drought stress. Addition of $\mathrm{H}_{2} / \mathrm{CO}$ significantly increased RWC during rooting under drought stress, resulting in higher adventitious root number. $\mathrm{CO}$ could regulate water balance and enhance salt tolerance in wheat seedling roots (Xie et al., 2008); HRW treatment also promoted RWC in M. sativa and improved its drought tolerance (Jin et al., 2016). Our results also indicated that $\mathrm{H}_{2} / \mathrm{CO}$ might promote adventitious rooting under drought stress through maintaining water holding capacity of plants. Moreover, $\mathrm{Hb} / \mathrm{ZnPPIX}$ reversed the effects of $\mathrm{H}_{2} / \mathrm{CO}$ on RWC, suggesting $\mathrm{CO}$ is involved in water balance induced by $\mathrm{H}_{2}$ during adventitious root development under drought stress.

Our results suggest that $\mathrm{HRW}, \mathrm{CO}$, and $\mathrm{CO}+\mathrm{HRW}$ significantly increased the contents of chl a, chl b, and chl $\mathrm{a}+\mathrm{b}$. Previous studies showed that $\mathrm{H}_{2}$ increased chlorophyll content and alleviated ultraviolet-B-triggered oxidative damage in $M$. sativa (Xie et al., 2015). Moreover, the application of CO increased chlorophyll content in wheat leaf and promoted wheat seed germination under drought stress (Liu et al., 2010). Liao et al. (2012) demonstrated that the enhanced chlorophyll content could effectively mitigate the adverse effect of drought stress in marigold and promoted adventitious rooting. Therefore, $\mathrm{H}_{2}$ and $\mathrm{CO}$ enhanced leaf chlorophyll content and then increased photosynthesis during drought and promoted adventitious rooting. In our study, drought stress led to the decrease in Fv/Fm, ФPSII and qP. However, HRW, CO, and CO+HRW alleviated the negative effect of drought on Fv/Fm, ФPSII, and qP. The result was consistent with the findings of Zhang et al. (2015) who reported that $\mathrm{H}_{2}$ enhanced Fv/Fm, ФPSII and qP in high light stress in maize. Zhang et al. (2012) reported that $\mathrm{CO}$ induced advantageous effects on the attenuation of salt-stress inhibition in Cassia obtusifolia seeds and seedlings. Our results indicated that HRW, CO, and $\mathrm{CO}+\mathrm{HRW}$ induced increase in $F \mathrm{v} / F \mathrm{~m}$, $\Phi$ PSII, and $\mathrm{qP}$, and promoted adventitious rooting under drought condition. In our experiment, when $\mathrm{Hb}$ or ZnPPIX was added to HRW/CO, the positive effect of HRW/CO was reversed. This demonstrates that $\mathrm{CO}$ might be involved in $\mathrm{H}_{2}$-regulated chlorophyll content and chlorophyll fluorescence parameters during adventitious rooting under drought stress.

The levels of TBARS, $\mathrm{O}_{2}{ }^{-}$, and $\mathrm{H}_{2} \mathrm{O}_{2}$ could reflect the degree of oxidative damage to membrane lipids. In the present study, the TBARS level was significantly increased under drought stress. However, the TBARS levels were significantly decreased in HRW, $\mathrm{CO}$, and $\mathrm{CO}+\mathrm{HRW}$ treatments. Higher lipid peroxidation has also been reported in salt stress-sensitive rice varieties (DionisioSese and Tobita, 1998). Cui et al. (2013) reported that the addition of HRW at $10 \%$ saturation significantly decreased contents of TBARS, and inhibited the appearance of Cd toxicity symptoms in alfalfa plants. Drought stress remarkably increased the TBARS level, but CO significantly decreased the TBARS content in plants subjected to salinity stress (Huang et al., 2006). Thus, it showed that $\mathrm{H}_{2}$ and $\mathrm{CO}$ could alleviate lipid peroxidation caused by drought stress. Also, our results suggested that drought stress resulted in a significant increase in the levels of $\mathrm{O}_{2}^{-}$and $\mathrm{H}_{2} \mathrm{O}_{2}$, and $\mathrm{HRW}, \mathrm{CO}$, and $\mathrm{CO}+\mathrm{HRW}$ could reverse it, showing that the drought stress-induced damage to cucumber explants had been alleviated. Jin et al. (2013) made a similar observation and reported that alfalfa seedlings pretreated with $0.39 \mathrm{mM} \mathrm{H}_{2}$ exhibited remarkable alleviation of oxidative damage. The exposure of alfalfa seedlings to $\mathrm{HgCl}_{2}$ triggered the production of reactive oxygen species (ROS), stunted growth and increased lipid peroxidation. However, such negative effects were obviously blocked by $\mathrm{H}_{2}$ (Cui et al., 2014). In another study, $\mathrm{CO}$ significantly decreased the contents of $\mathrm{O}_{2}{ }^{-}$and $\mathrm{H}_{2} \mathrm{O}_{2}$ in Indian mustard under Hg stress (Meng et al., 2011). Our experiment revealed that $\mathrm{HRW}, \mathrm{CO}$, and $\mathrm{CO}+\mathrm{HRW}$ enhanced drought tolerance in cucumber explants by decreasing TBARS, $\mathrm{O}_{2}{ }^{-}$, and $\mathrm{H}_{2} \mathrm{O}_{2}$ content. Moreover, when $\mathrm{Hb}$ or $\mathrm{ZnPPIX}$ were added to HRW/CO pretreatments, the positive effects of HRW/CO were reversed. Under $\mathrm{Cu}$ toxicity, the increased lipid peroxidation and contents of ROS had previously been reported in the mountain ginseng adventitious roots (Rajesh et al., 2008). These results suggested that $\mathrm{H}_{2}$ and $\mathrm{CO}$ could effectively mitigate the damage of drought stress via decreasing the levels of TBARS, $\mathrm{O}_{2}{ }^{-}$, and $\mathrm{H}_{2} \mathrm{O}_{2}$ during adventitious rooting under drought condition.

Plants possess an efficient antioxidant system to adjust to oxidative damage from biotic and abiotic stresses. Upon exposure to abiotic stresses, tolerant cells activate their antioxidant enzymatic system, which starts quenching the ROS and then protecting the cell (Wang and Liao, 2016). We found that the activities of SOD, POD, CAT, and APX were reduced under drought stress. HRW, CO, and CO+HRW treatments relieved the decrease of antioxidant enzymatic under drought stress. However, HRW, CO, and $\mathrm{CO}+\mathrm{HRW}$ treatments increased the activities of SOD, POD, CAT, and APX in cucumber adventitious rooting under drought stress. Studies have showed that $\mathrm{H}_{2}$ alleviated salinity stress in rice, which is associated with the induction of SOD, CAT, and APX (Xu et al., 2013). Cui et al. (2013) observed that $\mathrm{H}_{2}$ exhibited beneficial effects on the activities of SOD, POD, and APX and enhanced Cd stress tolerance in $M$. sativa. Moreover, Hemin and $\mathrm{CO}$ aqueous solution dose-dependently enhanced the activities of CAT and SOD, and alleviated the inhibition of seed germination and seedling growth under salt stress (Liu et al., 2007). CO increased the activities of SOD, POD, CAT, and APX, and it alleviated the inhibition of seed germination in C. obtusifolia L. and seedling growth under salinity stress (Zhang et al., 2012). Also, we found that $\mathrm{H}_{2}$ and $\mathrm{CO}$ improved drought tolerance in cucumber largely by increasing activities of SOD, POD, CAT, and APX. Meanwhile, $\mathrm{Hb}$ and ZnPPIX inhibited the promoted effects of HRW/CO under drought stress. The increase of POD activity has been known to be a rooting signal during root primordium formation (Ann-Caroline et al., 1991). There is a close relationship between the antioxidant enzymes and the formation of adventitious roots (Liao et al., 2010). The evidence from our study supported the hypothesis that under our experimental conditions, $\mathrm{H}_{2}$ and $\mathrm{CO}$ promoted adventitious root development under drought stress, and $\mathrm{CO}$ was involved in $\mathrm{H}_{2}$-inducedadventitious rooting under drought stress.

The changes in water soluble carbohydrate content may be linked to external environment. In the present study, we found that drought resulted in decreased content of water 
soluble carbohydrate. However, the application of HRW, CO, and $\mathrm{CO}+\mathrm{HRW}$ treatments to cucumber explants subjected to drought stress increased water soluble carbohydrate content. Indeed, $\mathrm{CO}$ was able to promote the activities of amylase and induced the formation of water soluble carbohydrate, thus resulting in the alleviation of oxidative damage caused by salt stress in Oryza sativa (Liu et al., 2007). Previous study showed that Hemin and CO enhanced the contents of total water soluble carbohydrate, soluble protein, and proline in C. obtusifolia L. leaf under salinity stress, which are contribute in improving plants resistance to stress (Zhang et al., 2012). Together, these results suggested that $\mathrm{H}_{2}$ and $\mathrm{CO}$ inhibited the decrease in water soluble carbohydrate content and subsequently promoted rooting under drought stress. Our study also showed that the soluble protein and proline accelerated the decline during rooting under drought condition. Soluble protein content was affected in tomato exposed to salt stress (Zeynep et al., 2010). CO modulated the accumulation of proline and increased tolerance in Indian mustard in $\mathrm{Hg}$ stress (Meng et al., 2011). In our experiment $\mathrm{HRW}, \mathrm{CO}$, and $\mathrm{CO}+\mathrm{HRW}$ treatments enhanced soluble protein and proline content. The levels of water soluble carbohydrate, soluble protein, and proline should be important considerations in the study of adventitious root development (Klerk, 1996). According to Zavattieri et al. (2009), the application of exogenous carbon sources (sucrose or glucose) was beneficial for the rooting of microshoots of Pinus pinea. Maintaining the levels of soluble carbohydrate and protein promoted adventitious rooting in marigold explants (Liao et al., 2012). Similarly, our results confirmed the importance of $\mathrm{CO}$ in $\mathrm{H}_{2}$-induced adventitious root development under drought stress. Thus, the results have an important functional implication, expanding and enriching the possibilities for $\mathrm{H}_{2}$ and CO during adventitious root development under various stresses.

\section{REFERENCES}

Ann-Caroline, N., Fernando, A. J., and Lennart, E. (1991). Effect of exogenous indole-3-acetic acid and indole-3-butyric acid on internal levels of the respective auxins and their conjugation with aspartic acid during adventitious root formation in pea cuttings. Plant Physiol. 96, 856-861. doi: 10.1104/pp.96. 3.856

Arnon, D. I. (1949). Copper enzymes in isolated chloroplasts: phenol oxidase in B. Vulgaris. Plant Physiol. 24, 1-15. doi: 10.1104/pp.24.1.1

Aroca, R., Vernieri, P., Irigoyen, J. J., Sánchez-Díaz, M., Tognoni, F., and Pardossi, A. (2003). Involvement of abscisic acid in leaf and root of maize (Zea mays L.) in avoiding chilling-induced water stress. Plant Sci. 165, 671-679. doi: 10.1016/S0168-9452(03)00257-7

Bates, L. S., Waldran, R. P., and Teare, I. D. (1973). Raipid determination of free proline for water studies. Plant Soil 39, 205-208. doi: 10.1007/BF00018060

Bradford, M. M. (1976). A rapid and sensitive method for the quantitation of microgram quantities of protein utilizing the principle of proteindye binding. Anal. Biochem. 72, 248-254. doi: 10.1016/0003-2697(76) 90527-3

Cao, Z. Y., Huang, B. K., Wang, Q. Y., Xuan, W., Ling, T. F., Zhang, B., et al. (2007). Involvement of carbon monoxide produced by heme oxygenase in ABAinduced stomatal closure in Vicia faba and its proposed signal transduction pathway. Chin. Sci. Bull. 52, 2365-2373. doi: 10.1007/s11434-007-0358-y

Chazen, O., Hartung, W., and Neumann, P. M. (1995). The different effects of PEG 6000 and $\mathrm{NaCI}$ on leaf development are associated with differential inhibition
The present study provides new insights into the roles and interactions of $\mathrm{H}_{2}$ and $\mathrm{CO}$ in their regulation of adventitious root development in cucumber seedlings under drought stress. We, therefore, conclude that $\mathrm{H}_{2}$ and $\mathrm{CO}$ the development of adventitious roots in cucumber seedlings by alleviating the negative effects of drought on RWC, chlorophyll content, chlorophyll fluorescence, antioxidant systems and osmotic strength, and inhibit oxidative damage of cucumber explants. Concurrently, the positive effect of $\mathrm{HRW} / \mathrm{CO}$ was reversed by $\mathrm{Hb}$ or $\mathrm{ZnPPIX}$, indicating that $\mathrm{CO}$ was involved in $\mathrm{H}_{2}$-induced adventitious root development in cucumber seedlings under drought stress.

\section{AUTHOR CONTRIBUTIONS}

WL designed the experiments; YC, MW and WL performed the experiments; YC, LH, MW and CL performed data analysis; YC and LH wrote the manuscript; WL and MD edited the manuscript.

\section{ACKNOWLEDGMENTS}

This work was supported by the National Natural Science Foundation of China (Nos. 31160398 and 31560563), the Post-Doctoral Foundation of China (Nos. 20100470887 and 2012T50828), the Key Project of Chinese Ministry of Education (No. 211182), the Research Fund for the Doctoral Program of Higher Education (No. 20116202120005), the Natural Science Foundation of Gansu References Province, China (Nos. 1606RJZA073, 1606RJZA077, 1308RJZA179, and 1308RJZA262) and Feitian and Fuxi Excellent Talents in Gansu Agricultural University in Lanzhou, P. R. China.

of root water transport. Plant Cell Environ. 18, 727-735. doi: 10.1111/j.13653040.1995.tb00575.x

Cui, W., Fang, P., Zhu, K. K., Mao, Y., Gao, C., Xie, Y. J., et al. (2014). Hydrogen-rich water confers plant tolerance to mercury toxicity in alfalfa seedlings. Ecotoxicol. Environ. Saf. 105, 103-111. doi: 10.1016/j.ecoenv.2014. 04.009

Cui, W., Gao, C., Fang, P., Lin, G. Q., and Shen, W. B. (2013). Alleviation of cadmium toxicity in Medicago sativa by hydrogen-rich water. J. Hazard. Mater. 260, 715-724. doi: 10.1016/j.jhazmat.2013.06.032

Cui, W. T., Qi, F., Zhang, Y. H., Cao, H., Zhang, J., Wang, R., et al. (2015). Methane-rich water induces cucumber adventitious rooting through heme oxygenase1/carbon monoxide and Ca2+ pathways. Plant Cell Rep. 34, 435-445. doi: $10.1007 / \mathrm{s} 00299-014-1723-3$

Dekker, J., and Hargrove, M. (2002). Weedy adaptation in Setaria spp. V. Effects of gaseous environment on giant foxtail (Setaria faberii) (Poaceae) seed germination. Am. J. Bot. 89, 410-416. doi: 10.3732/ajb.89.3.410

Dionisio-Sese, M. L., and Tobita, S. (1998). Antioxidant responses of rice seedlings to salinity stress. Plant Sci. 135, 1-9. doi: 10.1016/S0168-9452(98) 00025-9

Elstner, E. F., and Heupel, A. (1976). Inhibition of nitrite formation from hydroxylammoniumchloride: a simple assay for superoxide dismutase. Anal. Biochem. 70, 616-620. doi: 10.1016/0003-2697(76)90488-7

Farooq, M., Wahid, A., Kobayashi, N., Fujita, D., and Basra, S. M. A. (2009). Plant drought stress: effects, mechanisms and management. Agron. Sustain. Dev. 29, 185-212. doi: 10.1051/agro:2008021 
Heath, R. L., and Packer, I. (1968). Photoperoxidation in isolated chloroplst I, kinetics and stochiometry of fatty acid peroxidation. Arch. Biochem. Biophys. 125, 189-198. doi: 10.1016/0003-9861(68)90654-1

Hu, H. L., Li, P. X., Wang, Y. N., and Gu, R. X. (2014). Hydrogen-rich water delays postharvest ripening and senescence of kiwifruit. Food Chem. 156, 100-109. doi: 10.1016/j.foodchem.2014.01.067

Huang, B. K., Xu, S., Xuan, W., Li, M., Cao, Z. Y., Liu, K. L., et al. (2006). Carbon monoxide alleviates salt-induced oxidative damage in wheat seedling leaves. J. Integr. Plant Biol. 48, 249-254. doi: 10.1111/j.1744-7909.2006.00220.x

Jin, Q. J., Cui, W. T., Dai, C., Zhu, K. K., Zhang, J., Wang, R., et al. (2016). Involvement of hydrogen peroxide and heme oxygenase-1 in hydrogen gasinduced osomotic stress tolerance in alfalfa. Plant Growth Regul. 10, 215-223. doi: 10.1007/s10725-016-0159-x

Jin, Q. J., Zhu, K. K., Cui, W. T., Xie, Y. J., Han, B., and Shen, W. B. (2013). Hydrogen gas acts as a novel bioactive molecule in enhancing plant tolerance to paraquat-induced oxidative stress via the modulation of heme oxygenase1 signalling system. Plant Cell Environ. 36, 956-969. doi: 10.1111/pce. 12029

Klerk, G. D. (1996). Markers of adventitious root formation. Agronomie 16, 609-616. doi: 10.1051/agro:19961003

Liao, W. B., Huang, G. B., Yu, J. H., and Zhang, M. L. (2012). Nitric oxide and hydrogen peroxide alleviate drought stress in marigold explants and promote its adventitious root development. Plant Physiol. Biochem. 58, 6-15. doi: 10.1016/ j.plaphy.2012.06.012

Liao, W. B., Xiao, H. L., and Zhang, M. L. (2010). Effect of nitric oxide and hydrogen peroxide on adventitious root development from cuttings of groundcover chrysanthemum and associated biochemical changes. J. Plant Growth Regul. 29, 338-348. doi: 10.1007/s00344-010-9140-5

Lin, Y. T., Li, M. Y., Cui, W. T., Lu, W., and Shen, W. B. (2012). Haem oxygenase-1 is involved in hydrogen sulfide-induced cucumber adventitious root formation. J. Plant Growth Regul. 31, 519-528. doi: 10.1007/s00344-0129262-Z

Lin, Y. T., Zhang, W., Qi, F., Cui, W. T., Xie, Y. J., and Shen, W. B. (2014). Hydrogen-rich water regulates cucumber adventitious root development in a heme oxygenase-1/carbon monoxide-dependent manner. J. Plant Physiol. 171, 1-8. doi: 10.1016/j.jplph.2013.08.009

Ling, T. F., Zhang, B., Cui, W. T., Wu, M. Z., Lin, J. S., and Zhou, W. T. (2009). Carbon monoxide mitigates salt-induced inhibition of root growth and suppresses programmed cell death in wheat primary roots by inhibiting superoxide anion overproduction. Plant Sci. 177, 331-340. doi: 10.1016/j. plantsci.2009.06.004

Liu, K., Xu, S., Xuan, W., Ling, T. F., Cao, Z. Y., Huang, B. K., et al. (2007). Carbon monoxide counteracts the inhibition of seed germination and alleviates oxidative damage caused by salt stress in Oryza sativa. Plant Sci. 172, 544-555. doi: 10.1016/j.plantsci.2006.11.007

Liu, Y. H., Xu, S., Ling, T. F., Xu, L. L., and Shen, W. B. (2010). Heme oxygenase/carbon monoxide system participates in regulating wheat seed germination under osmotic stress involving the nitric oxide pathway. J. Plant Physiol. 167, 1371-1379. doi: 10.1016/j.jplph.2010.05.021

Meng, D. K., Chen, J., and Yang, Z. M. (2011). Enhancement of tolerance of Indian mustard (Brassica juncea) to mercury by carbon monoxide. J. Hazard. Mater. 186, 1823-1829. doi: 10.1016/j.jhazmat.2010.12.062

Mukherjee, S. P., and Choudhuri, M. A. (1983). Implications of water stressinduced changes in the levels of endogenous ascorbic acid and hydrogen peroxide in Vigna seedlings. Physiol. Plant. 58, 166-170. doi: 10.1111/j.13993054.1983.tb04162.x

Niu, L. J., and Liao, W. B. (2016). Hydrogen peroxide signaling in plant development and abiotic responses: crosstalk with nitric oxide and calcium. Front. Plant Sci. 7:230. doi: 10.3389/fpls.2016.00230

Ohsawa, I., Ishikawa, M., Takahashi, K., Watanabe, M., Nishimaki, K., Yamagata, K., et al. (2007). Hydrogen acts as a therapeutic antioxidant by selectively reducing cytotoxic oxygen radicals. Nat. Med. 13, 688-694. doi: $10.1038 / \mathrm{nm} 1577$

Piantadosi, C. A. (2002). Biological chemistry of carbon monoxide. Antioxid. Redox Signal. 4, 259-270. doi: 10.1089/152308602753666316

Rajesh, K. T., Eun-Joo, H., and Kee-Yoeup, P. (2008). Function of nitric oxide and superoxide anion in the adventitious root development and antioxidant defence in Panax ginseng. Plant Cell Rep. 27, 563-573. doi: 10.1007/s00299-0070448-y

Renwick, G. M., Giumarro, C., and Siegel, S. M. (1964). Hydrogen metabolism in higher plants. Plant Physiol. 39, 303-306. doi: 10.1104/pp.39. 3.303

She, X. P., and Song, X. G. (2008). Carbon monoxide-induced stomatal closure involves generation of hydrogen peroxide in Vicia faba guard cells. J. Integr. Plant Biol. 50, 1539-1548. doi: 10.1111/j.1744-7909.2008.00716.x

Siegel, S. M., Renwick, G., and Rosen, L. A. (1962). Formation of carbon monoxide during seed germination and seedling growth. Science 137, 683-684. doi: 10. 1126/science.137.3531.683

Smart, D. R., Kocsis, L., Walker, M. A., and Stockert, C. (2003). Dormant buds and adventitious root formation by Vitis and other woody plants. J. Plant Growth Regul. 21, 296-314. doi: 10.1007/s00344-003-0001-3

Su, N. N., Wu, Q., Liu, Y. Y., Cai, J. T., Shen, W. B., Xia, K., et al. (2014). Hydrogen-rich water reestablishes ROS homeostasis but exerts differential effects on anthocyanin synthesis in two varieties of radish sprouts under UV-A irradiation. J. Agric. Food Chem. 62, 6454-6462. doi: 10.1021/jf501 9593

Takahashi, F., Sato-Nara, K., Kobayashi, K., Mitsuo, S., and Hitoshi, S. (2003). Sugar-induced adventitious roots in Arabidopsis seedlings. J. Plant Res. 116, 83-91.

Wang, M., and Liao, W. (2016). Carbon monoxide as a signaling molecule in plants. Front. Plant Sci. 7:572. doi: 10.3389/fpls.2016.00572

Wu, M. Z., Huang, J. J., Xu, S., Ling, T. F., Xie, Y. J., and Shen, W. B. (2010). Haem oxygenase delays programmed cell death in wheat aleurone layers by modulation of hydrogen peroxide metabolism. J. Exp. Bot. 62, 235-248. doi: $10.1093 / \mathrm{jxb} / \mathrm{erq} 261$

Wu, Q., Su, N. N., Cai, J. T., Shen, Z. G., and Cui, J. (2015). Hydrogen-rich water enhances cadmium tolerance in Chinese cabbage by reducing cadmium uptake and increasing antioxidant capacities. J. Plant Physiol. 175, 174-182. doi: 10.1016/j.jplph.2014.09.017

Xie, Y., Ling, T., Han, Y., Liu, K., Zheng, Q., Huang, L., et al. (2008). Carbon monoxide enhances salt tolerance by nitric oxide-mediated maintenance of ion homeostasis and up-regulation of antioxidant defence in wheat seedling roots. Plant Cell Environ. 31, 1864-1881. doi: 10.1111/j.1365-3040.2008.01888.x

Xie, Y. J., Mao, Y., Lai, D., Zhang, W., and Shen, W. B. (2012). H2 enhances Arabidopsis salt tolerance by manipulating ZAT10/12-mediated antioxidant defence and controlling sodium exclusion. PLoS ONE 7:e49800. doi: 10.1371/ journal.pone. 0049800

Xie, Y. J., Mao, Y., Zhang, W., Lai, D. W., Wang, Q. Y., and Shen, W. B. (2014). Reactive oxygen species-dependent nitric oxide production contributes to hydrogen-promoted stomatal closure in Arabidopsis. Plant Physiol. 165, 759-773. doi: 10.1104/pp.114.237925

Xie, Y. J., Wei, Z., Duan, X. L., Dai, C., Zhang, Y. H., Cui, W. T., et al. (2015). Hydrogen-rich water-alleviated ultraviolet-B-triggered oxidative damage is partially associated with the manipulation of the metabolism of (iso)flavonoids and antioxidant defence in Medicago sativa. Funct. Plant Biol. 42, $1141-1157$.

Xu, S., Sa, Z. S., Cao, Z. Y., Xuan, W., Huang, B. K., Ling, T. F., et al. (2006). Carbon monoxide alleviates wheat seed germination inhibition and counteracts lipid peroxidation mediated by salinity. J. Integr. Plant Biol. 48, 1168-1176. doi: $10.1111 / j .1744-7909.2006 .00337 . x$

Xu, S., Zhu, S. S., Jiang, Y. L., Wang, N., Wang, R., Shen, W. B., et al. (2013). Hydrogen-rich water alleviates salt stress in rice during seed germination. Plant Soil 370, 47-57. doi: 10.1007/s11104-013-1614-3

Xuan, W., Zhu, F. Y., Xu, S., Huang, B. K., Ling, T. F., Qi, J. Y., et al. (2008). The heme oxygenase/carbon monoxide system is involved in the auxin-induced cucumber adventitious rooting process. Plant Physiol. 148, 881-893. doi: 10. 1104/pp.108.125567

Yannarelli, G. G., Noriega, G. O., Batlle, A., and Tomaro, M. L. (2006). Hemeoxygenase up-regulation in ultraviolet-B irradiated soybean plants involves reactive oxygen species. Planta 224, 1154-1162. doi: 10.1007/s00425006-0297-x

Zavattieri, A., Lima, M., Sobral, V., Oliveira, P., and Costa, A. (2009). Effects of carbon source, carbon concentration and culture conditions on in vitro rooting of Pinus pinea L. Microshoots. Acta Hortic. 812:173-180. 
Zeng, J., Zhang, M., and Sun, X. (2013). Molecular hydrogen is involved in phytohormone signaling and stress responses in plants. PLoS ONE 8:e71038. doi: 10.1371 /journal.pone. 0071038

Zeynep, B. D., Koksal, D., Hakan, B., and Ismail, G. (2010). Effects of salt stress on pigment and total soluble protein contents of three different tomato cultivars. Afr. J. Agric. Res. 5, 2056-2065.

Zhang, C. P., Li, Y. C., Yuan, F. G., Hu, S. J., and He, P. (2012). Effects of hematin and carbon monoxide on the salinity stress responses of Cassia obtusifolia L. seeds and seedlings. Plant Soil 359, 85-105. doi: 10.1007/s11104-0121194-7

Zhang, X. N., Zhao, X. Q., Wang, Z. Q., Shen, W. B., and Xu, X. M. (2015). Protective effects of hydrogen-rich water on the photosynthetic apparatus of maize seedlings (Zea mays L.) as a result of an increase in antioxidant enzyme activities under high light stress. Plant Growth Regul. 77, 43-56. doi: 10.1007/ s10725-015-0033-2

Zheng, Q., Meng, Q., Wei, Y. Y., and Yang, Z. M. (2011). Alleviation of copperinduced oxidative damage in chlamydomonas reinhardtii by carbon monoxide. Arch. Environ. Contam. Toxicol. 61, 220-227. doi: 10.1007/s00244-010-9602-6
Zhu, Y. C., Liao, W. B., Niu, L. J., Wang, M., and Ma, Z. J. (2016a). Nitric oxide is involved in hydrogen gas-induced cell cycle activation during adventitious root formation in cucumber. BMC Plant Biol. 16:146. doi: 10.1186/s12870-0160834-0

Zhu, Y. C., Liao, W. B., Wang, M., Niu, L. J., Xu, Q. Q., and Jin, X. (2016b). Nitric oxide is required for hydrogen gas-induced adventitious root formation in cucumber. J. Plant Physiol. 195, 50-58. doi: 10.1016/j.jplph.2016.02.018

Conflict of Interest Statement: The authors declare that the research was conducted in the absence of any commercial or financial relationships that could be construed as a potential conflict of interest.

Copyright $\odot 2017$ Chen, Wang, Hu, Liao, Dawuda and Li. This is an open-access article distributed under the terms of the Creative Commons Attribution License (CC BY). The use, distribution or reproduction in other forums is permitted, provided the original author(s) or licensor are credited and that the original publication in this journal is cited, in accordance with accepted academic practice. No use, distribution or reproduction is permitted which does not comply with these terms. 